Rev. Int. Contam. Ambie. 37, 89-107, 2021

https://doi.org/10.20937/RICA.53681

\title{
APLICACIÓN DE DOS METODOLOGÍAS PARA LA EVALUACIÓN DE LA CALIDAD DEL AGUA DE CUERPOS SUPERFICIALES DESTINADOS A LA PESCA EN EL SUR DEL ESTADO DE VERACRUZ, MÉXICO
}

Water quality index of surface water bodies for fishing in the south of the state of Veracruz, Mexico, applying two methodologies

\author{
Gabriela GARCÍA-RODRÍGUEZ ${ }^{1 *}$, Martha Elvira SANDOVAL-ROJAS ${ }^{1}$, \\ Ernesto Ramón CORONA-ROMANO ${ }^{1}$, Rosa Erika DE JESÚS-DE LA ROSA ${ }^{1}$, \\ Benito MARTÍNEZ-ROMAY ${ }^{1}$, Esther GARCÍA-GIL ${ }^{1}$ y Edith GARCÍA-GIL ${ }^{1}$
}

${ }^{1}$ Tecnológico Nacional de México, Instituto Tecnológico de Minatitlán, Blvd. Institutos Tecnológicos s/n, Colonia Buena Vista Norte, 96848 Minatitlán, Veracruz, México.

*Autor para correspondencia: gabygarcia_r@hotmail.com

(Recibido: julio de 2019; aceptado: junio de 2020)

Palabras clave: índice de calidad del agua, eutrofización, valores de referencia.

\begin{abstract}
RESUMEN
Se realizó la evaluación de la calidad del agua para protección de la vida acuática en 16 puntos de muestreo de cuerpos superficiales ubicados en una región entre Jáltipan y Texistepec, Veracruz, México. El estudio se realizó durante el periodo de estiaje y se aplicaron dos metodologías para evaluar la calidad del agua. Se seleccionaron los parámetros de caracterización: $\mathrm{pH}$, temperatura, oxígeno disuelto (OD), conductividad específica, turbidez, sólidos disueltos totales (SDT), sólidos suspendidos totales (SST), cloruros, dureza, alcalinidad, demanda bioquímica de oxígeno $\left(\mathrm{DBO}_{5}\right)$, demanda química de oxígeno (DQO), fósforo total, nitrógeno total Kjeldahl (NTK), nitrógeno orgánico $(\mathrm{N}-\mathrm{Org})$, nitrógeno de amonios $\left(\mathrm{N}-\mathrm{NH}_{4}^{+}\right)$, nitrógeno de nitritos $\left(\mathrm{N}^{-} \mathrm{NO}_{2}^{-}\right)$y nitrógeno de nitratos $\left(\mathrm{N}_{-} \mathrm{NO}_{3}{ }^{-}\right)$. La primera metodología es una modificación de la propuesta por la National Sanitation Foundation (NSF) de los EU, basada en ocho parámetros: OD, $\mathrm{pH}$, temperatura, $\mathrm{DBO}_{5}, \mathrm{SDT}$, turbidez, $\mathrm{N}_{-} \mathrm{NO}_{3}$ y fósforo total; la segunda está basada en la propuesta por la CNA. De esta metodología se contemplaron 13 parámetros de los 18 originales. Se obtuvieron índices de calidad del agua para cada punto estudiado, observándose que la calidad fluctuó de contaminada a excelente de acuerdo con los resultados alcanzados. En comparación con los Criterios Ecológicos de Calidad del Agua para protección de la vida acuática, los parámetros que más afectan la calidad del agua por incumplir las recomendaciones son $\mathrm{OD}$, fósforo total y N-NH${ }_{4}{ }^{+}$. Un parámetro que no se incluye en las metodologías utilizadas es la DQO; si se considera la Escala de Clasificación del Agua de Conagua conforme a la DQO, la cual es menos exigente que la normatividad internacional, los valores alcanzados por este parámetro en la zona de estudio clasifican al agua de contaminada a fuertemente contaminada.
\end{abstract}

Key words: water quality index, eutrophication, water quality reference values. 


\begin{abstract}
The evaluation of water quality for protection of aquatic life from surface waters was carried out in 16 sampling points in a region between Jáltipan and Texistepec, Veracruz, Mexico, during a dry season, applying two methodologies. The characterization parameters were $\mathrm{pH}$, temperature, dissolved oxygen (DO), conductivity, turbidity, total dissolved solids (TDS), total suspended solids (TSS), chlorides, hardness, alkalinity, biochemical oxygen demand $\left(\mathrm{BOD}_{5}\right)$, chemical oxygen demand (COD), total phosphorus, total Kjeldahl nitrogen (TKN), organic nitrogen, ammonium nitrogen $\left(\mathrm{N}_{-} \mathrm{NH}_{4}{ }^{+}\right)$, nitrite nitrogen $\left({\mathrm{N}-\mathrm{NO}_{2}}^{-}\right)$and nitrate nitrogen $\left(\mathrm{N}^{-} \mathrm{NO}_{3}{ }^{-}\right)$. The first methodology is a modification of the proposed by the US National Sanitation Foundation (NSF), which is based on eight parameters: $\mathrm{DO}, \mathrm{pH}$, temperature, $\mathrm{BOD}_{5}, \mathrm{TDS}$, turbidity, $\mathrm{N}-\mathrm{NO}_{3}$ and total phosphorus; while the second is based on the one proposed by CNA, which originally considers 18 parameters (only 13 were contemplated). From the application of both methodologies, water quality indexes were obtained for each point studied, observing that the quality fluctuated from contaminated to excellent. Contrasting with the Ecological Criteria of Water Quality for the protection of aquatic life, the parameters that most affect the quality of water for noncompliance with the recommendations are DO, total phosphorus and $\mathrm{N}_{-} \mathrm{NH}_{4}{ }^{+}$. A parameter that is not included in the methodologies used is the COD. According to the Water Quality Criteria from Conagua (less demanding than international regulations), the values reached for COD in the study area classify the water from contaminated to heavily contaminated.
\end{abstract}

\section{INTRODUCCIÓN}

El agua es un recurso que desempeña un papel fundamental como componente abiótico dentro de los ecosistemas naturales, como elemento vital para el consumo humano y como factor básico para su uso y aprovechamiento sostenible en los sectores económico, productivo y de servicios (López-Álvarez et al. 2013, Azpilcueta-Pérez et al. 2017).

El concepto calidad del agua se refiere al conjunto de características físicas, químicas y biológicas clasificadas en relación con características modelo definidas según el uso del recurso (Rubio-Arias et al. 2012). Así, la evaluación de la calidad del agua puede definirse como la evaluación de las características físicas, químicas y biológicas del agua con relación a su estado natural, efectos antropogénicos y usos futuros, siguiendo ciertos estándares establecidos por diversas entidades nacionales o internacionales (Balmaseda-Espinosa y García-Hidalgo 2014, Quiroz-Fernández et al. 2017).

Las actividades antrópicas generadoras de efluentes urbanos, agrícolas e industriales, aunadas a los procesos naturales de formación y modificación de los suelos, originan alteraciones en la calidad de los cuerpos receptores de agua (Espinal-Carreón et al. 2013). Estas fluctuaciones de calidad no sólo son estacionales - por periodo de lluvias o estiaje - sino también espaciales, lo cual representa un reto cuando se pretende diagnosticar las tendencias en la calidad de un cuerpo de agua (De la Mora-Orozco et al. 2004, Tyagi et al. 2013).

La mayoría de los cuerpos superficiales del país reciben descargas de aguas residuales sin tratamiento, lo que ocasiona niveles variables de contaminación que limitan el uso directo del agua, ya que se requieren diferentes calidades. De esta contaminación no están exentos los acuíferos, que pueden recibir emisiones contaminantes por infiltración a través de los diversos horizontes del suelo.

En este sentido, desde 1989 se establecieron en México los Criterios Ecológicos de la Calidad del Agua CE-CCA-001/89 (SEDUE 1989), los cuales fijan valores que se consideran aptos o recomendados para parámetros relevantes de acuerdo con el uso al que se destine el agua.

El modo más conveniente para expresar la calidad de los recursos hídricos es el Índice de Calidad del Agua (ICA) con el uso de datos de caracterización, el cual es útil para modificar las políticas de gestión de recursos hídricos. Un ICA reduce la información procedente de un número de parámetros físicos, químicos y biológicos usados en el monitoreo de la calidad del agua a una única expresión, con el objetivo de facilitar la interpretación de dicha información y permitir el acceso público a datos sobre la calidad del agua (Mădălina y Breabăn 2014).

Esta metodología fue desarrollada por la Fundación Nacional de Saneamiento (NSF-National Sanitation Foundation) en Estados Unidos (Brown et al. 
1970) y mejorada por Deininger para la Academia Nacional de Ciencias de Estados Unidos (NAS 1975).

En México, el ICA desarrollado y aplicado por la Comisión Nacional del Agua (CNA, hoy Conagua) en la década de 1970, permite clasificar el agua por sus características de calidad. El ICA es un valor en una escala de 0 a 100 que indica el nivel de contaminación de un cuerpo de agua (un mayor valor de ICA indica una mejor calidad del agua); se obtiene a partir de un promedio ponderado de los índices de calidad individuales de 18 parámetros físicos, químicos y biológicos, dentro de los que se encuentran el pH, la demanda bioquímica de oxígeno $\left(\mathrm{DBO}_{5}\right)$ y los sólidos suspendidos totales (SST) (SEMARNAT 2019).

En las últimas décadas el crecimiento poblacional y principalmente el crecimiento industrial han impactado los cuerpos de agua con descargas, las cuales vierten contaminantes tóxicos tales como metales pesados y compuestos orgánicos que no estaban considerados en el ICA, motivo por el cual la evaluación con dicho índice en la actualidad es parcial y no necesariamente corresponde a la realidad (SEMARNAT 2019). Por tanto, se ha recurrido al establecimiento de Criterios de Clasificación de la Calidad del Agua conforme a la demanda bioquímica de oxígeno $\left(\mathrm{DBO}_{5}\right)$, la demanda química de oxígeno (DQO) y los sólidos suspendidos totales (SST), con lo cual se generan escalas de calidad del agua que van de excelente a fuertemente contaminada, con criterios intermedios de buena calidad, aceptable y contaminada (CONAGUA 2010). En 2017 se añadieron cinco indicadores más al análisis de la calidad del agua superficial: coliformes fecales (CF), Escherichia coli, enterococos, porcentaje de saturación de oxígeno (OD \%) y toxicidad (TOX) (CONAGUA 2019).

Sin embargo, a la fecha se considera que el ICA es una herramienta práctica y útil para la evaluación, comparación y comunicación de la calidad de cuerpos de agua, ya que engloba de manera simplificada parámetros indicadores de deterioro en los mismos, lo cual muchas veces se vuelve complicado cuando se dispone de un número elevado de datos. Diversos investigadores han realizado numerosos estudios para evaluar la calidad de cuerpos superficiales y subterráneos en varias partes del mundo, a través de varias metodologías: Yogendra y Puttaiah (2008), Reza y Singh (2010) y Batabyal y Chakraborty (2015) en la India; Olguín et al. (2010), Guzmán-Colis et al. (2011) y Espinal-Carreón et al. (2013) en México; Espejo et al. (2012) en Chile; Balmaseda-Espinosa y García-Hidalgo (2014) en Cuba; Mădălina y Breabăn (2014) en Rumania, y Quiroz-Fernández et al. (2017) en Ecuador, quienes han identificado el nivel de contaminación en reservorios, lagos, ríos y agua subterránea.

Fernández y Solano (2005) afirman que en el mundo hay por lo menos 30 índices de calidad de agua que son de uso común, y consideran un número de variables que van de tres a 72. Prácticamente todos estos índices incluyen al menos tres de los siguientes parámetros: oxígeno disuelto (OD), $\mathrm{DBO}_{5}$ o DQO, nitrógeno en forma amoniacal y de nitratos $\left(\mathrm{NH}_{4}-\mathrm{N}\right.$ y N-NO 3 , fósforo en forma de ortofosfato $\left(\mathrm{PO}_{4}-\mathrm{P}\right)$, $\mathrm{pH}$ y sólidos totales (ST) o SST (Samboni-Ruiz et al. 2007). En la actualidad, en la evaluación de la calidad del agua hay tendencia a incluir indicadores bióticos que la definen de acuerdo con los efectos sobre la vida acuática (e.g. los macroinvertebrados bentóni$\cos$ ), estudiando su diversidad y abundancia frente a alteraciones en su ecosistema (Asadollahfardi 2015, Mosquera-Murillo y Mosquera-Mosquera 2017).

En los diversos arroyos, esteros y ríos que circundan a los municipios de Texistepec, Jáltipan, Cosoleacaque, Oteapan y Chinameca, en el sur del estado de Veracruz, México, la pesca era hasta hace unos años una de las actividades económicas más importantes; sin embargo, el deterioro en la calidad de los cuerpos de agua superficiales ha mermado drásticamente la producción obtenida por las diversas cooperativas pesqueras de la zona, las cuales manifestaron su preocupación e interés en la realización de esta investigación. En este contexto, se evaluó la calidad del agua de arroyos tributarios y esteros contiguos al río Chiquito, ubicados en el sur del estado de Veracruz, mediante la aplicación de dos metodologías para determinar el índice de calidad del agua.

\section{MATERIALES Y MÉTODOS}

Se eligieron 16 puntos de muestreo a lo largo de arroyos y esteros que confluyen en el río Chiquito, afluente del río Coatzacoalcos. Todos los puntos de muestreo corresponden a la Subcuenca Hidrográfica RH29Ba-R Coatzacoalcos y se encuentran distribuidos dentro de los municipios de Cosoleacaque, Chinameca, Oteapan, Jáltipan y Texistepec. Durante el recorrido de inspección inicial se pudo apreciar vida silvestre propia de la región. También se observaron áreas de cultivo de maíz, melón, chile habanero y calabaza.

Los criterios para seleccionar los puntos de muestreo fueron:

a. Presencia de actividades agroindustriales con el fin de investigar vertidos no controlados. 
b. Disminución de la producción pesquera en ciertas corrientes superficiales (de acuerdo con información proporcionada por pescadores de la región).

c. Deterioro apreciable de la calidad de los cuerpos de agua de la zona (presencia de malos olores y crecimiento de lirio acuático, entre otros aspectos).

El muestreo en los 16 puntos seleccionados se realizó en la primera quincena de mayo de 2018, de acuerdo con la Norma Mexicana NMX-AA-014-1980 (SECOFI 1980). Las condiciones generales fueron de día nublado a soleado. Se identificaron tres posibles descargas clandestinas: una proveniente de una industrializadora de maíz nixtamalizado (Descarga A, punto 1); una procedente de una refinadora de aceite de palma crudo (Descarga B, punto 1A) y una correspondiente a una procesadora de alimento para aves (Descarga C, punto 2), las tres ubicadas en el municipio de Chinameca.

Los puntos 3 y 5 fueron ubicados en el arroyo Ocosoapan, en tanto que los puntos 4 y 6 corresponden a Ojo de Agua y al estero Mozapan, respectivamente. Los puntos 7, 8, 9 y 10 se encuentran sobre el río Chiquito. El punto 11 corresponde a un canal que recibe escorrentías agrícolas de una zona de cultivo de palma de aceite (conocido como Compuerta La Palmera), el cual descarga hacia el Canal de Navegación (punto 12), contiguo a la presa Agua de Minas. El punto 13 está ubicado sobre el arroyo Correa a la altura del puente Monte Negro y confluye en el punto 14. El punto 14 se ubica en la confluencia de las corrientes del estero Mozapan, del Canal de Navegación, arroyo Correa y Laguna de Préstamos, el cual se identifica como bocana del Estero Aztapan y Canal de Navegación. El punto 15 corresponde a un cuerpo de agua conocido como Laguna de Préstamos, ubicado al sureste de la presa Agua de Minas y conectado con el punto 14. Finalmente, el punto 16 se ubica en el arroyo Apepecho, afluente del estero Aztapan y adyacente a la presa Agua de Minas.

El cuadro I presenta las coordenadas geográficas de los 16 puntos de muestreo seleccionados para la evaluación de la calidad del agua. Asimismo, la delimitación de la zona de estudio se muestra en la figura 1, donde se observan las conexiones existentes entre el río Chiquito y los arroyos y esteros que lo circundan.

Los parámetros físicos y químicos analizados fueron: temperatura, $\mathrm{pH}, \mathrm{OD}$, conductividad específica, turbidez, sólidos en todas sus formas, cloruros, alcalinidad, dureza total, $\mathrm{DBO}_{5}, \mathrm{DQO}, \mathrm{NTK}, \mathrm{N}^{-\mathrm{NH}_{4}}{ }^{+}$, nitrógeno orgánico, $\mathrm{N}-\mathrm{NO}_{2}, \mathrm{~N}_{-} \mathrm{NO}_{3}$ y fósforo total. Para la determinación analítica de los parámetros mencionados se aplicaron las Normas Mexicanas (NMX) basadas en APHA-AWWA-WPCF (1992).

CUADRO I. PUNTOS DE MUESTREO SELECCIONADOS Y COORDENADAS GEOGRÁFICAS DE LA ZONA ENTRE JÁLTIPAN Y TEXISTEPEC, VERACRUZ.

\begin{tabular}{|c|c|c|c|}
\hline $\begin{array}{l}\text { Puntos de } \\
\text { muestreo }\end{array}$ & Descripción del punto & Latitud norte & Longitud oeste \\
\hline Punto $1^{*}$ & Industria nixtamalizadora de maíz & $18^{\circ} 0{ }^{\prime} 30.48^{\prime \prime} \mathrm{N}$ & $94^{\circ} 41^{\prime} 18.74^{\prime \prime} \mathrm{O}$ \\
\hline Punto $1 A^{*}$ & Procesadora de producto refinado de aceite de palma & $18^{\circ} 0 \prime 43.34 ” \mathrm{~N}$ & $94^{\circ} 41^{\prime} 0.96^{\prime \prime} \mathrm{O}$ \\
\hline Punto $2 *$ & Procesadora de alimento para aves & $18^{\circ} 0 ’ 21.04 ” \mathrm{~N}$ & $94^{\circ} 41^{\prime} 34.09^{\prime \prime} \mathrm{O}$ \\
\hline Punto 3 & Arroyo Ocosoapan (a la altura de Coacotla, Ver.) & $17^{\circ} 56^{\prime} 31.70^{\prime \prime} \mathrm{N}$ & $94^{\circ} 40^{\prime} 49.58^{\prime \prime} \mathrm{O}$ \\
\hline Punto 4 & Ojo de Agua (a la altura del rancho Caxiapan) & $17^{\circ} 53^{\prime} 59.99^{\prime \prime} \mathrm{N}$ & $94^{\circ} 40^{\prime} 50.03^{\prime \prime} \mathrm{O}$ \\
\hline Punto 5 & Arroyo Ocosoapan (antes de llegar a Estero Mozapan) & $17^{\circ} 53^{\prime} 18.69^{\prime \prime} \mathrm{N}$ & $94^{\circ} 41^{\prime} 15.83^{\prime \prime} \mathrm{O}$ \\
\hline Punto 6 & Estero Mozapan & $17^{\circ} 53 ’ 18.22^{\prime} " \mathrm{~N}$ & $94^{\circ} 41^{\prime} 17.73{ }^{\prime \prime} \mathrm{O}$ \\
\hline Punto 7 & Río Chiquito 1 & $17^{\circ} 52^{\prime} 54.39^{\prime \prime} \mathrm{N}$ & $94^{\circ} 40^{\prime} 49.82^{\prime \prime} \mathrm{O}$ \\
\hline Punto 8 & Río Chiquito 2 & $17^{\circ} 52^{\prime} 20.84^{\prime \prime} \mathrm{N}$ & $94^{\circ} 40^{\prime} 0.32^{\prime \prime} \mathrm{O}$ \\
\hline Punto 9 & Río Chiquito (Paso La Victoria) & $17^{\circ} 52^{\prime} 12.67^{\prime \prime} \mathrm{N}$ & $94^{\circ} 38^{\prime} 55.71^{\prime \prime} \mathrm{O}$ \\
\hline Punto 10 & Río Chiquito (Lomas de Tacamichapan) & $17^{\circ} 52^{\prime} 4.32^{\prime} \mathrm{N}$ & $94^{\circ} 42^{\prime} 15.57^{\prime \prime} \mathrm{O}$ \\
\hline Punto 11 & Compuerta La Palmera & $17^{\circ} 52^{\prime} 22.61^{\prime \prime} \mathrm{N}$ & $94^{\circ} 45^{\prime} 13.17^{\prime \prime} \mathrm{O}$ \\
\hline Punto 12 & Canal de Navegación & $17^{\circ} 52^{\prime} 24.37^{\prime \prime} \mathrm{N}$ & $94^{\circ} 45^{\prime} 11.91 ” \mathrm{O}$ \\
\hline Punto 13 & Arroyo Correa (Puente Montenegro) & $17^{\circ} 51^{\prime} 29.96 ” \mathrm{~N}$ & $94^{\circ} 44^{\prime} 12.15^{\prime \prime} \mathrm{O}$ \\
\hline Punto 14 & Bocana del Estero Aztapan y Canal de Navegación & $17^{\circ} 52^{\prime} 22.17^{\prime \prime} \mathrm{N}$ & $94^{\circ} 44^{\prime} 6.79^{\prime \prime} \mathrm{O}$ \\
\hline Punto 15 & Laguna de Préstamos & $17^{\circ} 52^{\prime} 29.95^{\prime} \mathrm{N}$ & $94^{\circ} 44^{\prime} 1.85^{\prime \prime} \mathrm{O}$ \\
\hline Punto 16 & Arroyo Apepecho & $17^{\circ} 52^{\prime} 47.76^{\prime \prime} \mathrm{N}$ & $94^{\circ} 44^{\prime} 0.04^{\prime \prime} \mathrm{O}$ \\
\hline
\end{tabular}

*Puntos en los cuales no se determinó el ICA. 


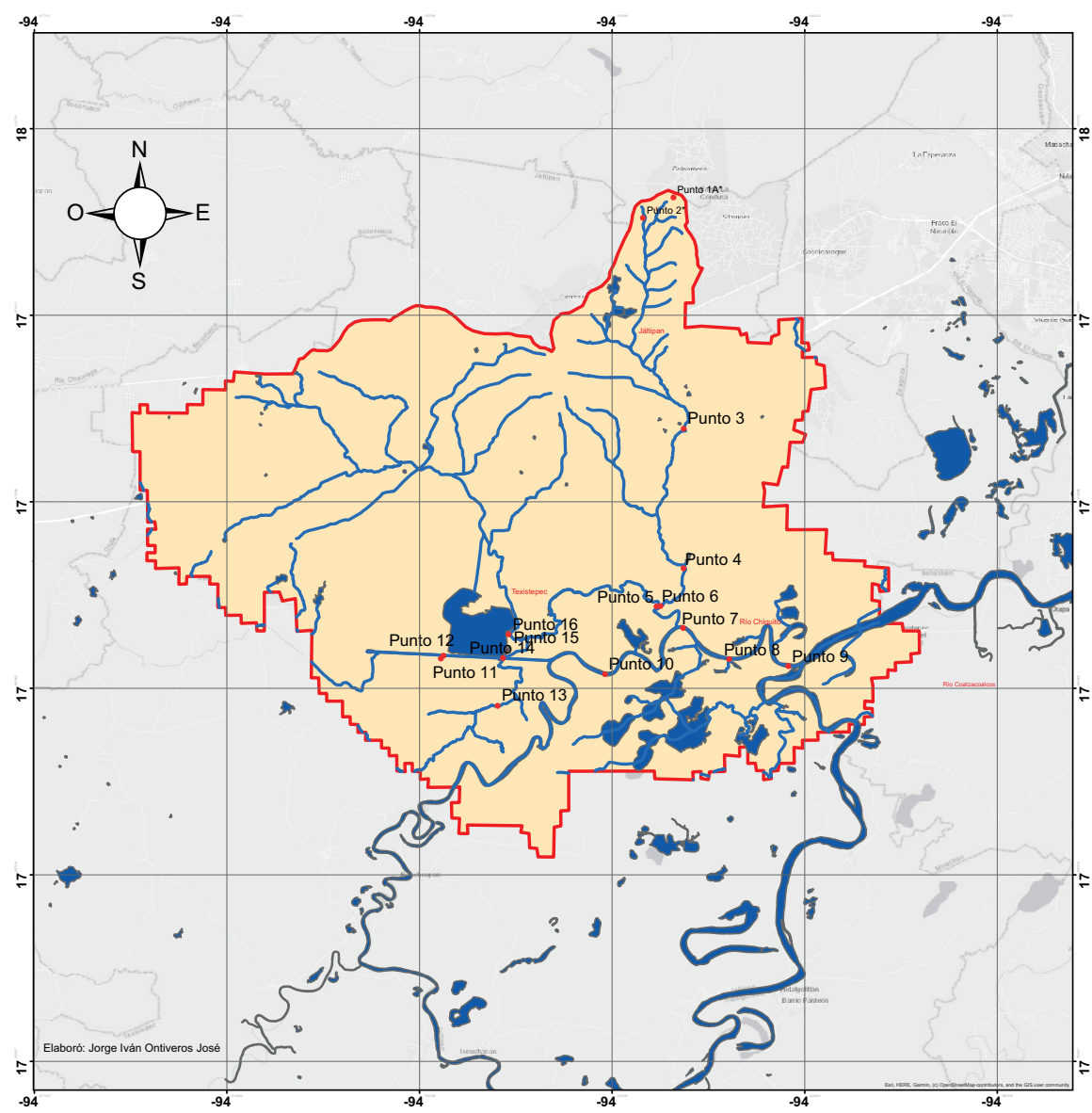

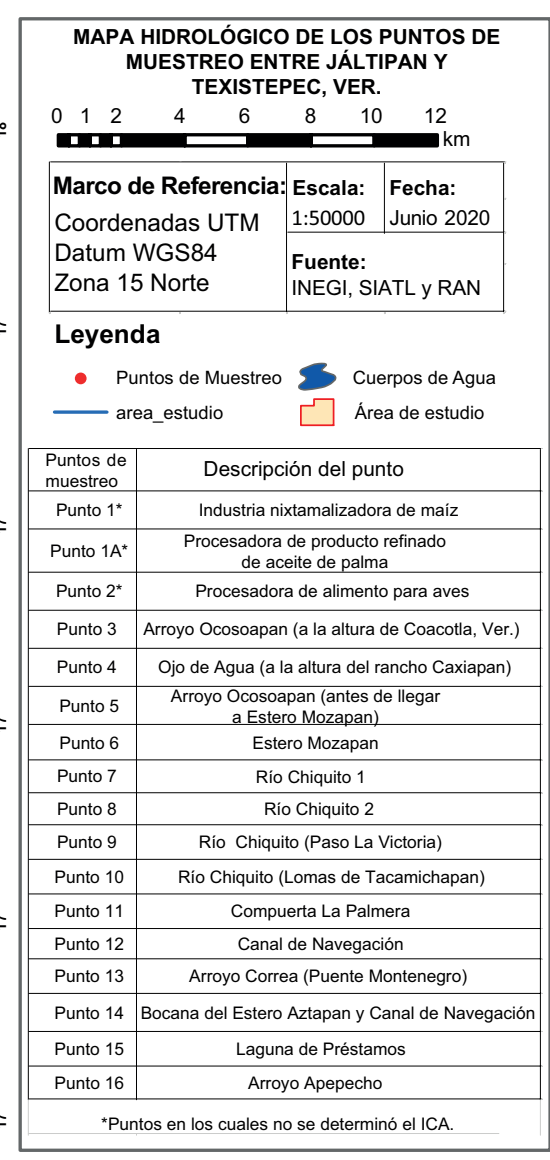

Fig. 1. Mapa hidrológico de los puntos de muestreo entre Jáltipan y Texistepec, Veracruz.

Los análisis se efectuaron en el Laboratorio de Investigaciones del Medio Ambiente (LIMA) del Instituto Tecnológico de Minatitlán, utilizando los métodos mostrados en el cuadro II.

Los cuerpos de agua de interés se hallan en una zona donde el principal uso del recurso hídrico es la pesca. Por tal motivo, y por el objetivo mismo de la investigación, se evaluó la calidad del agua para protección de la vida acuática, mediante la aplicación de dos metodologías.

\section{Metodología de cálculo ICA-NSF}

ICA-NSF es una metodología muy práctica que surge en la década de 1970. En la actualidad se utiliza para supervisar la calidad de los ríos a través del tiempo y comparar aguas de abastecimiento en Estados Unidos y muchos países del mundo (NSF 2019). En su desarrollo intervinieron 142 expertos en el tema de calidad del agua, quienes usaron la técnica de investigación Delphi, seleccionando los nueve parámetros que consideraron más relevantes: $\mathrm{DBO}_{5}, \mathrm{OD}$, coliformes fecales, $\mathrm{N}-\mathrm{NO}_{3}, \mathrm{pH}$, cambio de temperatura, sólidos disueltos totales (SDT), fósforo total y turbiedad. Se asignaron los pesos relativos o pesos de importancia del parámetro (Wi) correspondientes a los factores de contaminación de aguas, de acuerdo con el uso del agua y la importancia de los parámetros en relación con el riesgo que implica el aumento o disminución de su concentración.

Para calcular el ICA-NSF se usa una suma lineal ponderada de los subíndices ( $\mathrm{ICA}_{\mathrm{a}}$, promedio aritmético ponderado, ecuación 1) o una función de agregación del producto ponderado ( $\mathrm{ICA}_{\mathrm{m}}$, función ponderada multiplicativa, ecuación 2), considerando los pesos asignados a cada variable, que son: OD, 0.17 ; coliformes fecales, $0.15 ; \mathrm{pH}, 0.12 ; \mathrm{DBO}_{5}$, $0.10 ; \mathrm{N}^{-\mathrm{NO}_{3}}, 0.10$; fosfatos, 0.10 ; desviación de temperatura, 0.10 ; turbiedad, 0.08 , y SDT, 0.08 (Ott 1978).

$I C A_{a}=\sum_{i=1}^{9}\left(\operatorname{Sub}_{i} W_{i}\right)$

$C A_{m}=\prod_{i=1}^{9}\left(\operatorname{Sub}_{i}^{W_{i}}\right)$ 
CUADRO II. NORMAS TÉCNICAS UTILIZADAS PARA EVALUACIÓN DE PARÁMETROS.

\begin{tabular}{|c|c|c|}
\hline Parámetro & Norma técnica & Método utilizado \\
\hline Oxígeno disuelto & $\begin{array}{l}\text { NMX-AA-012-SCFI-2001. Determinación de Oxígeno Disuelto en } \\
\text { aguas naturales, residuales y residuales tratadas. }\end{array}$ & Método yodométrico \\
\hline $\begin{array}{l}\text { Nitrógeno total } \\
\text { Kjeldahl }\end{array}$ & $\begin{array}{l}\text { NMX-AA-026-SCFI-2010. Medición de Nitrógeno Total Kjeldahl en } \\
\text { aguas naturales, residuales y residuales tratadas. }\end{array}$ & Método semimicro Kjeldahl \\
\hline $\begin{array}{l}\text { Demanda bioquími- } \\
\text { ca de oxígeno }\end{array}$ & $\begin{array}{l}\text { - NMX-AA-028-SCFI-2001. Determinación de la Demanda Bioquímica } \\
\text { de Oxígeno en aguas naturales, residuales }\left(\mathrm{DBO}_{5}\right) \text { y residuales tratadas. }\end{array}$ & Método yodométrico \\
\hline Fósforo total & $\begin{array}{l}\text { NMX-AA-029-SCFI-2001. Determinación de fósforo total en aguas } \\
\text { naturales, residuales y residuales tratadas. }\end{array}$ & Método Cloruro estanoso \\
\hline $\begin{array}{l}\text { Demanda química } \\
\text { de oxígeno }\end{array}$ & $\begin{array}{l}\text { NMX-AA-030/1-SCFI-2012. Medición de la demanda química de oxíge- } \\
\text { no en aguas naturales, residuales y residuales tratadas }\end{array}$ & $\begin{array}{l}\text { - Método espectrofotométrico } \\
\text { de reflujo cerrado }\end{array}$ \\
\hline $\begin{array}{l}\text { Sólidos en todas } \\
\text { sus formas }\end{array}$ & $\begin{array}{l}\text { NMX-AA-034-SCFI-2015. Medición de sólidos y sales disueltas en } \\
\text { aguas naturales, residuales y residuales tratadas }\end{array}$ & Método gravimétrico \\
\hline $\begin{array}{l}\text { Acidez y } \\
\text { alcalinidad }\end{array}$ & $\begin{array}{l}\text { NMX-AA-036-SCFI-2001. Determinación de acidez y alcalinidad en } \\
\text { aguas naturales, residuales y residuales tratadas }\end{array}$ & Método volumétrico \\
\hline Turbiedad & $\begin{array}{l}\text { NMX-AA-038-SCFI-2001. Determinación de turbiedad en aguas natura- } \\
\text { les, residuales y residuales tratadas }\end{array}$ & - Métod \\
\hline Dureza & $\begin{array}{l}\text { NMX-AA-072-SCFI-2001. Determinación de dureza total en aguas } \\
\text { naturales, residuales y residuales tratadas }\end{array}$ & Método volumétrico \\
\hline Cloruros totales & $\begin{array}{l}\text { NMX-AA-073-SCFI-2001. Determinación de cloruros totales en } \\
\text { aguas naturales, residuales y residuales tratadas }\end{array}$ & $\begin{array}{l}\text { Método volumétrico } \\
\text { Argentometría }\end{array}$ \\
\hline Nitratos & $\begin{array}{l}\text { NMX-AA-079-SCFI-2001. Determinación de nitratos en aguas natura- } \\
\text { les, potables, residuales y residuales tratadas }\end{array}$ & Método de sulfato de brucina \\
\hline $\begin{array}{l}\text { Conductividad } \\
\text { específica }\end{array}$ & $\begin{array}{l}\text { NMX-AA-093-SCFI-2000. Determinación de la conductividad } \\
\text { específica }\end{array}$ & Método electrométrico \\
\hline Nitritos & $\begin{array}{l}\text { NMX-AA-099-SCFI-2006. Determinación de nitrógeno de nitritos } \\
\text { en aguas naturales y residuales }\end{array}$ & Método colorimétrico \\
\hline
\end{tabular}

donde $S u b_{i}$ es el subíndice del parámetro $i$ y $W_{i}$ es el factor de ponderación del parámetro $i$. El resultado obtenido se interpreta de acuerdo con el cuadro III. En la actualidad, el ICA-NSF original se ha modificado para desarrollar ICA específicos para determinadas regiones o países.

CUADRO III. ESCALA DE CLASIFICACIÓN DEL ICANSF.

\begin{tabular}{lcc}
\hline Calidad & Rango & Escala de color \\
\hline Excelente & $91-100$ & Azul \\
Buena & $71-90$ & Verde \\
Media & $51-70$ & Amarillo \\
Mala & $26-50$ & Naranja \\
Muy mala & $0-25$ & Rojo \\
\hline
\end{tabular}

En esta investigación se realizó una modificación del modelo desarrollado por la NSF consistente en la reducción del número original de parámetros de 9 a 8 :
$\mathrm{OD}, \mathrm{pH}, \mathrm{DBO}_{5}, \mathrm{~N}_{-} \mathrm{NO}_{3}{ }^{-}$, fósforo total, temperatura, SDT y turbidez. A pesar de que los coliformes fecales tienen un peso ponderado de 0.15 (el segundo en importancia de acuerdo con la NSF), este parámetro no se consideró como variable crítica para los cuerpos de agua estudiados debido a que en el cálculo del ICA se considera un uso para protección de la vida acuática, no como fuente de abastecimiento para consumo humano.

El cálculo del ICA se realizó con el promedio aritmético ponderado mediante la ecuación 3 :

$I C A=\sum_{i=1}^{8} S I_{i} W_{i}$

Fue necesario ajustar el peso ponderado de cada parámetro individualmente, repartiendo equitativamente el peso de la variable eliminada del ICA desarrollado por NSF entre los ocho parámetros restantes, para tener un peso ponderado final de 1.0, como se muestra en el cuadro IV.

Para la normalización del subíndice de cada variable se utilizaron curvas de función (Brown et al. 1970, 
CUADRO IV. PESOS PONDERADOS (Wi) DE LOS PARÁMETROS EMPLEADOS EN EL CÁLCULO DEL ICA, COMPARÁNDOLOS CON LOS ORIGINALES DE LANSF.

\begin{tabular}{lcc}
\hline Párametro & $\mathrm{W}_{\mathrm{i}}$ (NSF) & $\mathrm{W}_{\mathrm{i}}$ \\
\hline Oxígeno disuelto & 0.17 & 0.18875 \\
Coliformes fecales & 0.15 & $\mathrm{NC}$ \\
$\mathrm{pH}$ & 0.12 & 0.13875 \\
$\mathrm{DBO}_{5}$ & 0.10 & 0.11875 \\
$\mathrm{~N}^{-} \mathrm{NO}_{3}^{-}$ & 0.10 & 0.11875 \\
Fósforo total & 0.10 & 0.11875 \\
Temperatura & 0.10 & 0.11875 \\
Sólidos disueltos totales & 0.08 & 0.09875 \\
Turbidez & 0.08 & 0.09875 \\
Total & 1.0 & 1.0 \\
\hline
\end{tabular}

NC: no considerado para el cálculo del ICA

Quiroz-Fernández et al. 2017) estimadas a partir de las medias aritméticas con un límite de confianza de $80 \%$ sobre el valor medio. Estos ajustes se hicieron de acuerdo con la metodología establecida por NSF.

Los resultados del ICA se clasificaron mediante la escala sugerida por House y Ellis (1987), la cual se muestra mediante un conjunto de colores en el cuadro III (Fernández y Solano 2005).

\section{Metodología de cálculo ICA-CNA}

De acuerdo con la CNA, la contaminación de un cuerpo de agua se representa por el índice de calidad del agua (ICA), que está expresado como un porcentaje del agua pura; así, el agua altamente contaminada tendrá un ICA global cercano o igual a $0 \%$, en cambio el agua en excelentes condiciones tendrá un valor cercano al $100 \%$. Con ayuda del ICA se pueden determinar los usos a los que se puede destinar el agua según su nivel de contaminación.

El ICA fue desarrollado en varias etapas. En la primera etapa se creó una escala de calificación acorde con los diferentes usos del agua. La segunda involucró el desarrollo de una escala de calificación para cada parámetro; para ello se emplearon los 18 tipos diferentes de indicadores ambientales mostrados en el cuadro $\mathbf{V}$ con el fin de establecer una correlación entre los diferentes parámetros y su influencia en la contaminación del recurso hídrico (SEMARNAT 2019).

Después de que se prepararon estas escalas se formularon modelos matemáticos para cada parámetro con el fin de convertir los datos físicos a un índice de calidad individual $\left(I_{X}\right)$ (Cuadro V). Estos $I_{X}$ se promedian para generar un índice compuesto $(I)$ de la muestra de agua (Gracia 2004).

CUADRO V. ECUACIONES PARA LOS DIFERENTES PARÁMETROS PROPUESTOS PARA EVALUAR EL ÍNDICE DE CALIDAD DEL AGUA.

\begin{tabular}{|c|c|c|c|}
\hline Parámetro & Fórmula & Unidades & Peso $(\mathrm{W})^{*}$ \\
\hline $\mathrm{pH}$ & $\begin{array}{l}\mathrm{I}_{\mathrm{Ph}}=10^{0.2335 \mathrm{Ph}+.044} \\
\mathrm{I}_{\mathrm{Ph}}=100 \\
\mathrm{I}_{\mathrm{Ph}}=10^{4.22-0.295}\end{array}$ & $\begin{array}{c}\text { Si } \mathrm{pH}<6.7 \\
\text { Si } 6.7>\mathrm{pH}<7.3 \\
\text { Si } \mathrm{pH}>7.3\end{array}$ & 1.0 \\
\hline Color (C) & $\mathrm{I}_{\mathrm{C}}=123 \mathrm{C}^{-0.295}$ & Unidades $\mathrm{Pt} / \mathrm{Co}$ & 1.0 \\
\hline Turbiedad (T) & $\mathrm{I}_{\mathrm{T}}=108(\mathrm{~T})^{-0.178}$ & UTJ & 0.5 \\
\hline Grasas y aceites & $\mathrm{I}_{\mathrm{GA}}=87.25(\mathrm{GA})^{-0.298}$ & $\mathrm{mg} / \mathrm{L}$ & 2.0 \\
\hline SST (SS) & $\mathrm{I}_{\mathrm{SS}}=266.5(\mathrm{SS})-0.37$ & $\mathrm{mg} / \mathrm{L}$ & 1.0 \\
\hline SDT (SD) & $\mathrm{I}_{\mathrm{SD}}=109.1-0.175(\mathrm{SD})$ & $\mathrm{mg} / \mathrm{L}$ & 0.5 \\
\hline Conductividad (CE) & $\mathrm{I}_{\mathrm{CE}}=540(\mathrm{CE})^{-0.379}$ & Siemen/cm & 2.0 \\
\hline Alcalinidad (A) & $\mathrm{I}_{\mathrm{A}}=105(\mathrm{~A})^{-0.186}$ & $\mathrm{mg} \mathrm{CaCO}_{3} / \mathrm{L}$ & 1.0 \\
\hline Dureza total (D) & $\mathrm{I}_{\mathrm{D}}=10^{1.974-0.00174 \mathrm{D}}$ & $\mathrm{mg} \mathrm{CaCO}_{3} / \mathrm{L}$ & 1.0 \\
\hline Nitratos $\left(\mathrm{NO}_{3}\right)$ & $\mathrm{I}_{\mathrm{NO} 3}=162.2(\mathrm{NO} 3)^{-0.343}$ & $\mathrm{mg} / \mathrm{L}$ & 2.0 \\
\hline Nitrógeno amoniacal, $\left(\mathrm{NH}_{3}\right)$ & $\mathrm{I}_{\mathrm{NH} 3}=45.8(\mathrm{NH} 3)^{-0.343}$ & $\mathrm{mg} / \mathrm{L}$ & 2.0 \\
\hline Fosfatos totales $\left(\mathrm{PO}_{4}\right)$ & $\mathrm{I}_{\mathrm{PO} 4}=34.215(\mathrm{PO} 4)^{-0.46}$ & $\mathrm{mg} / \mathrm{L}$ & 2.0 \\
\hline Cloruros $(\mathrm{Cl})$ & $\mathrm{I}_{\mathrm{Cl}}=121(\mathrm{Cl})^{0.233}$ & $\mathrm{mg} / \mathrm{L}$ & 0.5 \\
\hline Oxígeno disuelto (OD) & $\mathrm{I}_{\mathrm{OD}}=((\mathrm{OD}) /(\%$ Odsat $)) * 100$ & $\mathrm{mg} / \mathrm{L}$ & 5.0 \\
\hline $\mathrm{DBO}_{5}$ & $\mathrm{I}_{\mathrm{DBO}}=120(\mathrm{DBO})^{-0.673}$ & $\mathrm{mg} / \mathrm{L}$ & 5.0 \\
\hline Coliformes totales (CT) & $\mathrm{I}_{\mathrm{CT}}=97.5(\mathrm{CT})^{-0.27}$ & $\mathrm{NMP} / 100 \mathrm{~mL}$ & 3.0 \\
\hline Coliformes fecales (CF) & $\mathrm{I}_{\mathrm{CF}}=97.5(5(\mathrm{CF}))^{-0.27}$ & $\mathrm{NMP} / 100 \mathrm{Ml}$ & 4.0 \\
\hline Detergentes (DE) & $\mathrm{I}_{\mathrm{DE}}=100-16.67(\mathrm{DE})+0.1587(\mathrm{DE})^{2}$ & $\mathrm{mg} / \mathrm{L}$ & 3.0 \\
\hline
\end{tabular}

*Peso "W" asignado a cada parámetro de acuerdo con el grado de afectación de éstos sobre la calidad en el agua. 
Ciertos parámetros son más significativos que otros para la calidad del agua, por lo que fueron ponderados en orden de importancia. El peso $W$ al que se añadió el subíndice $i$ representa la ponderación, de modo que la fórmula que proporciona el ICA es la ecuación 4:

$I C A=\left[\frac{\sum_{i=1}^{n} I_{i} W_{i}}{\sum_{i=1}^{n} W_{i}}\right]$

donde: $I C A$ es el índice de calidad del agua, $I i$ es el índice de calidad del parámetro $i, W_{i}$ es el peso de importancia del parámetro $i$ y $n$ es el número de parámetros.

En el cuadro VI se presentan los valores de la Escala de Clasificación General de la Calidad de Agua, así como los criterios de clasificación.

En esta investigación se consideraron en total 13 parámetros: $\mathrm{pH}$, turbidez, $\mathrm{SST}$, SDT, cloruros, $\mathrm{OD}$, $\mathrm{DBO}_{5}$, conductividad específica, alcalinidad, dureza total, $\mathrm{N}-\mathrm{NH}_{4}, \mathrm{~N}_{-} \mathrm{NO}_{3}$ y fosfatos totales. No se consideraron los parámetros de color, grasas y aceites, coliformes totales y fecales, y sustancias activas al azul de metileno (SAAM), originalmente incluidos en el modelo de ICA-CNA, con el fin de priorizar otros indicadores de caracterización del agua para protección de la vida acuática más relevantes. Por tanto, en la ecuación 4 no se consideraron los subíndices de los parámetros eliminados ni su ponderación respectiva.

\section{RESULTADOS}

En el cuadro VII se presentan los valores obtenidos de los parámetros de caracterización fisicoquímica en cada punto del muestreo. En el caso de las tres posibles descargas clandestinas descubiertas, al momento del muestreo sólo se pudo obtener una muestra del vertido de la industrializadora de maíz nixtamalizado (punto 1), que descarga hacia un arroyuelo de escaso caudal en época de estiaje. En la zona de vertido de la procesadora de alimento para aves y de la refinadora de aceite de palma crudo, de características similares al área de descarga del punto 1, se hallaron residuos de materia orgánica, por lo que se deduce que existen descargas intermitentes.

En el punto 3 se percibió un ligero olor a descargas sanitarias y se observó la escorrentía de un potrero adyacente. Esta última situación fue notoria también en el punto 4. En los puntos de muestreo 5 al 9 sólo se observó un poco de lirio acuático en las orillas de los cauces de agua, y no se registró la presencia de otro factor indicativo de contaminación. En la zona comprendida de los puntos 10 al 16 se observó gran cantidad de lirio en las orillas de los cuerpos de agua,
CUADRO VI. USOS DEL AGUA SEGÚN EL ÍNDICE DE CALIDAD DEL AGUA.

\begin{tabular}{lll}
\multicolumn{3}{l}{ Agua potable } \\
\hline $90-100$ & E & No requiere purificación para consumo \\
$80-90$ & A & Purificación menor requerida \\
$70-80$ & LC & Dudoso su consumo sin purificación \\
$50-70$ & C & Potabilización necesaria \\
$40-50$ & FC & Dudosa para consumo \\
$0-40$ & EC & Inaceptable para consumo
\end{tabular}

Agrícola

90-100 E No requiere purificación para riego

70-90 A Purificación menor para cultivos hortícolas

50-70 LC Utilizable en la mayoría de los cultivos

30-50 C Requiere tratamiento a la mayoría de los cultivos

20-30 FC Solo para cultivos muy resistentes

0-20 EC Inaceptable para riego

Pesca y vida acuática

70-100 E Pesca y vida acuática abundante

60-70 A Límite para peces muy sensibles

50-60 LC Pesca dudosa con riesgos de salud

40-50 C Vida acuática para peces muy resistentes

30-40 FC Inaceptable para actividad pesquera

0-30 EC Inaceptable para vida acuática

\begin{tabular}{lll}
\hline Industrial & \\
\hline $90-100$ & E & No requiere purificación \\
$70-90$ & A & Purificación menor para que usen agua limpia \\
$50-70$ & LC & No requiere tratamiento para muchas industrias \\
$30-50$ & C & Tratamiento para la mayoría de los usos \\
$20-30$ & FC & Uso restringido en actividades burdas \\
$0-20$ & EC & Inaceptable para cualquier industria
\end{tabular}

Recreativo

70-100 E Cualquier tipo de deporte acuático

50-70 A Restringida para deporte de inmersión

40-50 LC Dudosa para el contacto con el agua

30-40 C Solo navegación

20-30 FC Contaminación visible, evitar cercanía

0-20 EC Inaceptable para recreación

por lo que fue difícil el acceso y se necesitó abrir paso a las canoas con remos y manos. Asimismo, se encontraron botes de agroquímicos flotando en las orillas de los cuerpos superficiales y algunos entre los cultivos.

En el punto 12 fue difícil efectuar el muestreo (Fig. 2), ya que estaba completamente cubierto por lirio acuático, probablemente debido a que en este sitio se reciben escorrentías agrícolas de una zona de cultivo de palma de aceite. De acuerdo con estudios reportados, esto puede ocasionar pérdida de organismos acuáticos, situación que se refleja en la desaparición de especies como la mojarra y el 


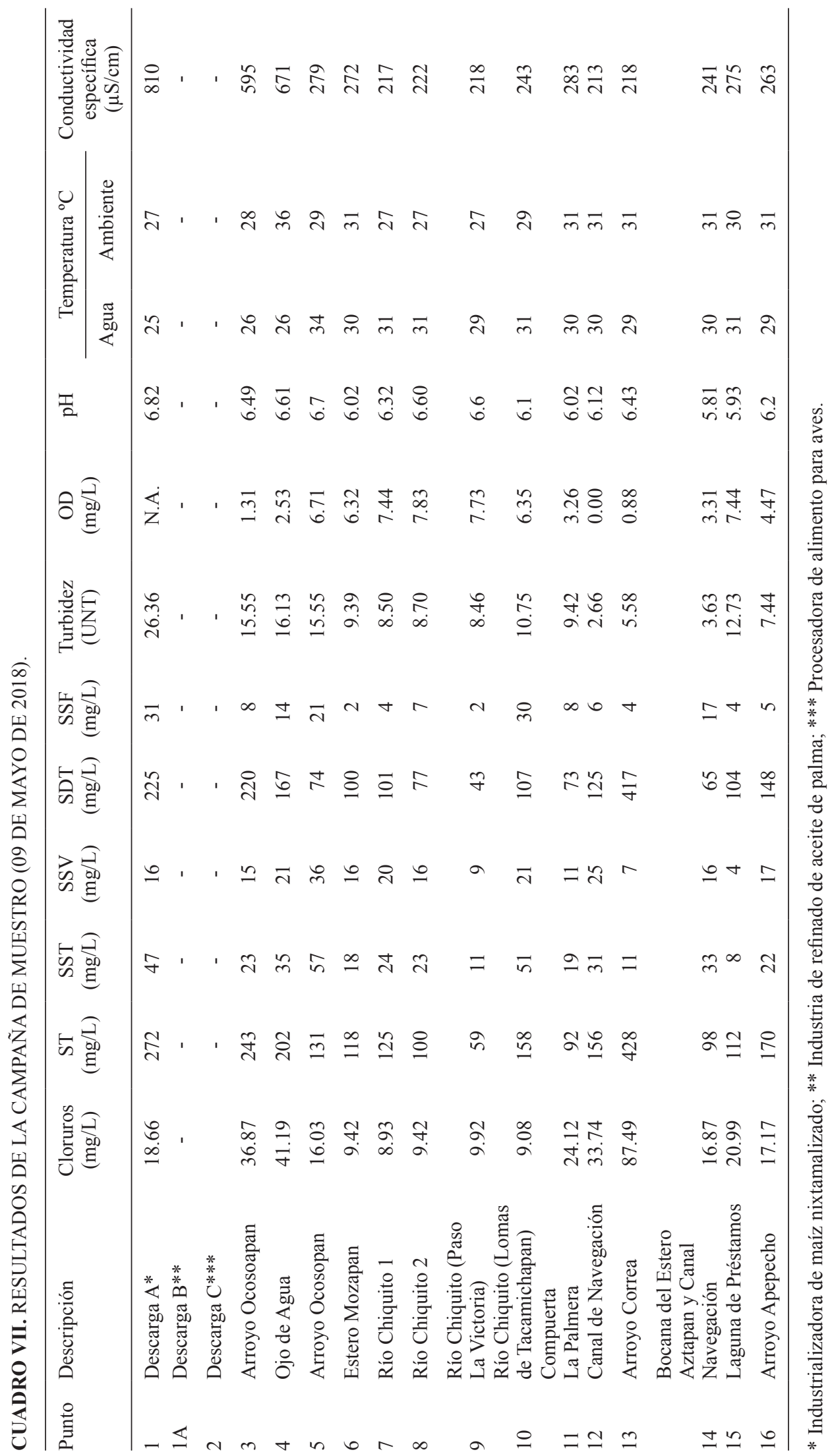




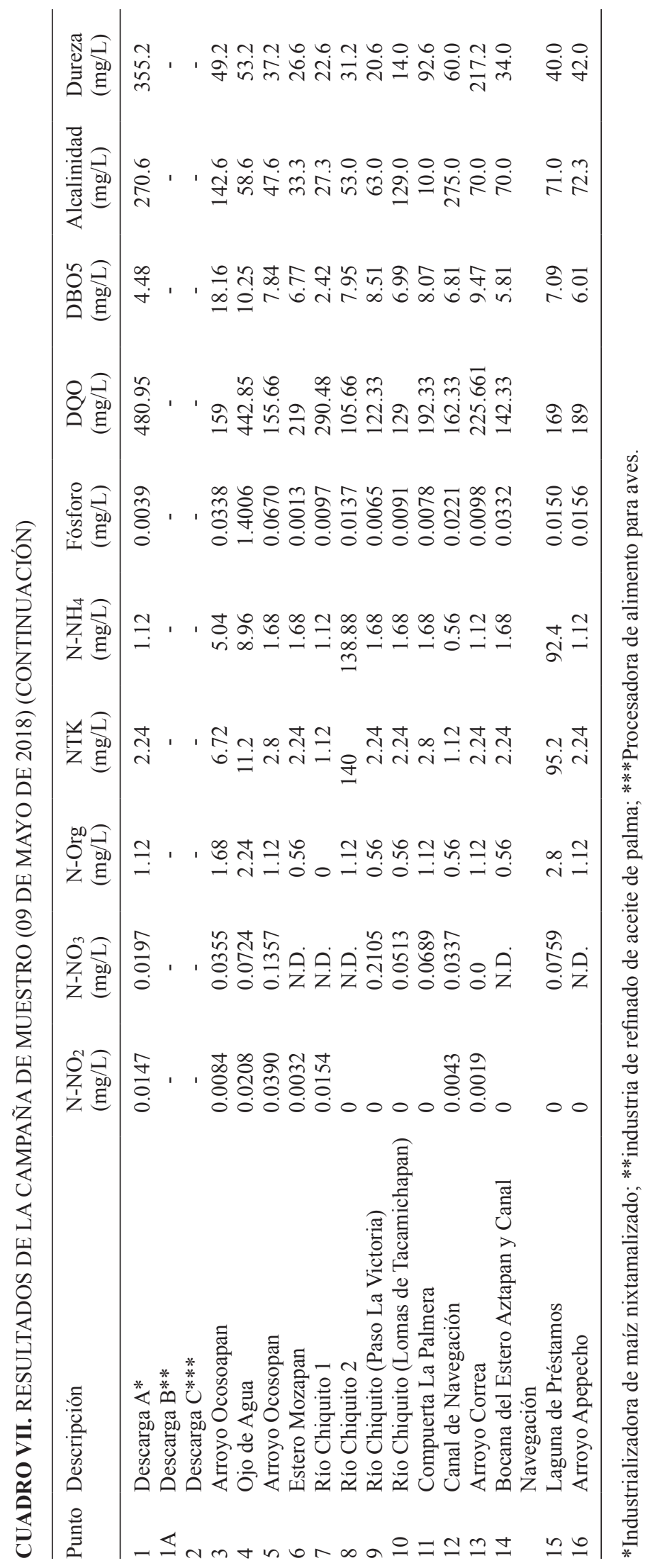




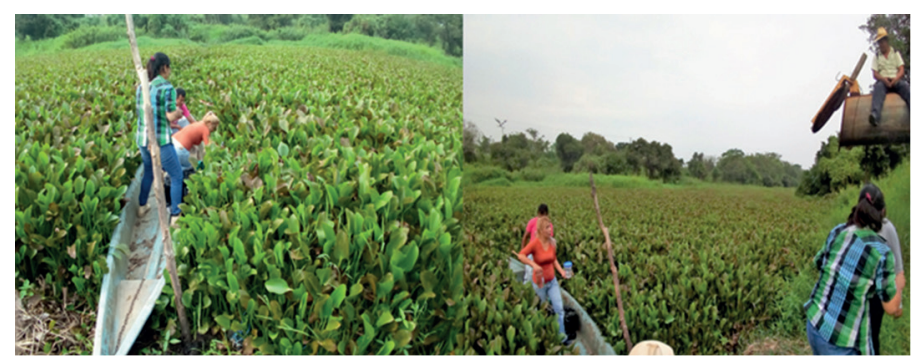

Fig. 2. Punto 12: Canal de Navegación, completamente cubierto de lirio acuático, en Texistepec, Veracruz.

pejelagarto, que son propias de la zona y abundan en condiciones propicias (Miller 2009).

De la muestra tomada en el punto 1 (Descarga A) sólo se realizó una caracterización fisicoquímica (cuadro VII), pero no se evaluó el ICA. Los parámetros más relevantes en la Descarga A fueron la conductividad específica de $810 \mu \mathrm{S} / \mathrm{cm}$ (que evidencia una cantidad considerable de sales en solución, quizás proveniente de la cal $[\mathrm{CaO}]$ que se utiliza para nixtamalizar el maíz), y la DQO con un valor de $480.95 \mathrm{mg} / \mathrm{L}$, en tanto que la $\mathrm{DBO}_{5}$ sólo fue de 4.48 $\mathrm{mg} / \mathrm{L}$. El valor de la DQO puede impactar la calidad de los cuerpos superficiales aguas abajo.

Un parámetro muy importante para evaluar la calidad de los cuerpos de agua para protección de la vida acuática es el OD. Este parámetro fluctuó entre los puntos 3 al 16 de 0 a $7.83 \mathrm{mg} / \mathrm{L}$, siendo los puntos $3,4,11,12,13,14$ y 16 donde se presentaron concentraciones inferiores a $5 \mathrm{mg} / \mathrm{L}$, límite mínimo recomendado por los Criterios Ecológicos de Calidad del Agua CE-CCA-001/89 (SEDUE 1989). Los puntos 3 y 4 podrían verse impactados por descargas de aguas residuales sin tratar procedentes de la comunidad de Coacotla (además de las escorrentías de potreros adyacentes), ya que la DQO alcanzó valores de 159 y $442.85 \mathrm{mg} / \mathrm{L}$, respectivamente.

La DQO en los puntos 3 al 16 varió de 105.66 a $442.85 \mathrm{mg} / \mathrm{L}$, niveles similares a los encontrados en aguas residuales urbanas (o industriales). En este sentido, la Escala de Clasificación de la Calidad del Agua conforme a la DQO (CONAGUA 2010) cataloga estas aguas como contaminadas a fuertemente contaminadas. La DBO varió de 2.42 a $18.16 \mathrm{mg} / \mathrm{L}$, clasificándose como aguas de calidad excelente a aceptable, de acuerdo con la Escala de Clasificación de la Calidad del Agua conforme a la $\mathrm{DBO}_{5}$ (CONAGUA 2010). Esta gran disparidad entre los valores de la DQO y la $\mathrm{DBO}_{5}$ en todos los puntos de muestreo permite establecer que la mayor demanda de oxígeno se debe a materia difícilmente biodegradable.
Otros parámetros importantes para la protección de la vida acuática son los concernientes a procesos de eutrofización. En este sentido, los Criterios Ecológicos de Calidad del Agua CE-CCA-001/89 (SEDUE 1989) establecen un criterio máximo recomendado de $0.06 \mathrm{mg} / \mathrm{L}$ para N-NH${ }_{4}{ }^{+}$, en tanto que para el fósforo total el límite máximo es de $0.1 \mathrm{mg} \mathrm{PO}_{4}^{-2} / \mathrm{L}$ en arroyos y ríos. Con relación al N-NH${ }_{4}^{+}$, éste se vio rebasado en todos los puntos de muestreo, fluctuando desde un valor mínimo de 0.56 a $138.88 \mathrm{mg} / \mathrm{L}$. La presencia de esta forma de nitrógeno pudiera provenir de la existencia de descargas sanitarias de algunas comunidades, de las excretas del ganado o de la aplicación de fertilizantes que, a pesar de ser época de estiaje, fluyen por escorrentías hacia los arroyos, ya que las aguas en algunos puntos muestreados se utilizan para riego. Respecto al fósforo total, sólo se rebasó el límite recomendado en el punto 4. Es conveniente mencionar que en algunos puntos en los que no se rebasaron los niveles de fósforo se observó, al momento del muestreo, presencia de lirio acuático (por ejemplo, en los puntos 11, 12 y 15). Esto indica que el fósforo presente en agua podría haber generado problemas de eutrofización, disminuyendo el OD disponible para los peces.

\section{DISCUSIÓN}

El cuadro VIII presenta los valores del ICA obtenidos en los 13 puntos de muestreo aplicando las metodologías del NSF y la CNA. Se incluye también la categorización de la calidad de acuerdo con cada metodología.

La figura 3 muestra la comparación de los ICA obtenidos en la campaña de muestreo mediante ambas metodologías, en tanto que las figuras 4 y 5 muestran la distribución espacial de la calidad del agua en la zona de estudio determinada mediante la aplicación de los índices NSF y CNA, respectivamente. 
CUADRO VIII. COMPARACIÓN DE LOS VALORES DE ICA OBTENIDOS APLICANDO LAS METODOLOGÍAS NSF Y CNA.

\begin{tabular}{|c|c|c|c|c|}
\hline \multirow{2}{*}{$\begin{array}{l}\text { Punto de } \\
\text { muestreo }\end{array}$} & \multicolumn{4}{|c|}{ Periodo de estiaje } \\
\hline & $\begin{array}{l}\text { ICA } \\
\text { NSF }\end{array}$ & Clasificación & $\begin{array}{l}\text { ICA } \\
\text { CNA }\end{array}$ & Clasificación \\
\hline 3 & 59.93 & $\begin{array}{l}\text { Medianamente } \\
\text { contaminada }\end{array}$ & 52.0 & $\begin{array}{l}\text { Levemente } \\
\text { contaminada }\end{array}$ \\
\hline 4 & 56.36 & $\begin{array}{l}\text { Medianamente } \\
\text { contaminada }\end{array}$ & 50.4 & $\begin{array}{l}\text { Levemente } \\
\text { contaminada }\end{array}$ \\
\hline 5 & 80.08 & Buena & 72.7 & Excelente \\
\hline 6 & 79.93 & Buena & 71.7 & Excelente \\
\hline 7 & 85.14 & Buena & 83.0 & Excelente \\
\hline 8 & 82.00 & Buena & 72.4 & Excelente \\
\hline 9 & 82.30 & Buena & 75.4 & Excelente \\
\hline 10 & 79.17 & Buena & 70.4 & Excelente \\
\hline 11 & 69.15 & $\begin{array}{l}\text { Medianamente } \\
\text { contaminada }\end{array}$ & 61.6 & Aceptable \\
\hline 12 & 64.64 & $\begin{array}{l}\text { Medianamente } \\
\text { contaminada }\end{array}$ & 53.6 & $\begin{array}{l}\text { Levemente } \\
\text { contaminada }\end{array}$ \\
\hline 13 & 61.52 & $\begin{array}{l}\text { Medianamente } \\
\text { contaminada }\end{array}$ & 53.8 & $\begin{array}{l}\text { Levemente } \\
\text { contaminada }\end{array}$ \\
\hline 14 & 71.37 & Buena & 62.5 & Aceptable \\
\hline 15 & 80.64 & Buena & 71.9 & Excelente \\
\hline 16 & 74.22 & Buena & 66.7 & Aceptable \\
\hline
\end{tabular}

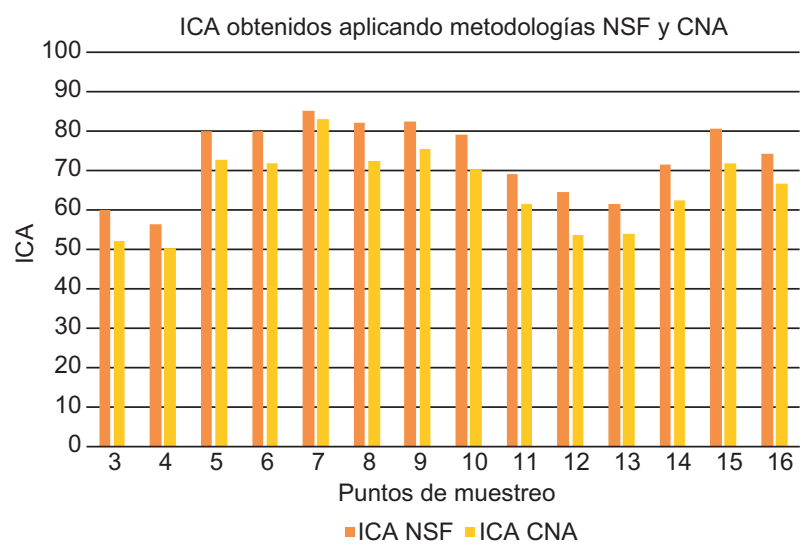

Fig. 3. Valores de ICA obtenidos aplicando la metodología de la NSF y la CNA.

De acuerdo con lo observado en el cuadro VIII, la metodología NSF clasifica la calidad del agua de medianamente contaminada a buena, en tanto que la calidad del agua varía de levemente contaminada a excelente según la metodología de la CNA. En esta última, los valores del ICA tienden a ser menores que los obtenidos con el NSF, ya que considera una mayor cantidad de parámetros; sin embargo, la clasificación de la calidad es menos exigente.
En términos generales, hay concordancia entre las evaluaciones de ambas metodologías. En los puntos 3 y 4, que reciben descargas de aguas residuales sanitarias y de escorrentías de ganado, se establece la calidad del agua contaminada, aunque las dos metodologías difieren en el nivel de contaminación (medianamente contaminada para el índice NSF y levemente contaminada de acuerdo con la CNA). Lo mismo sucede en los puntos 12 y 13. Por otra parte, las metodologías NSF y CNA clasifican el agua de los puntos 5 al 10 como de calidad buena y excelente, respectivamente. En el punto 11, el índice NSF clasifica el agua como medianamente contaminada, en tanto que el de la CNA la considera como aceptable. En los puntos 14, 15 y 16 estas metodologías clasifican el agua como buena (NSF) y de aceptable a excelente (CNA).

Una razón por la cual la metodología CNA pudiera ser menos estricta es que establece diferentes escalas para cada uso del agua (Cuadro VI), en este caso para protección de la vida acuática. La metodología del NSF es más general, aunque se han seleccionado los parámetros considerados prioritarios para la vida acuática.

Por ello es necesario analizar los parámetros más relevantes para el cálculo del ICA con cada metodología, para correlacionarlos con los valores obtenidos en éstas. Con fines de comparar los valores de referencia de calidad del agua en cuerpos superficiales para protección de la vida acuática establecidos en los Criterios Ecológicos de Calidad del Agua en México, se hizo una revisión de normativas internacionales que establecen rangos recomendables, seleccionándose los criterios que se muestran en el cuadro IX.

\section{Oxígeno disuelto}

Para ambas metodologías, los puntos en los cuales se cataloga el agua como buena y excelente (5 al 10) son aquellos donde existe un mayor caudal y movimiento de las aguas, lo cual propicia la autodepuración; en ellos, parámetros críticos para la vida acuática, como el OD, alcanzan valores superiores a $5 \mathrm{mg} / \mathrm{L}$ (en el rango de 6.32 a $7.83 \mathrm{mg} / \mathrm{L}$ ). Tal es el caso del Río Chiquito en sus dos puntos, Paso La Victoria y Lomas de Tacamichapan. No obstante, hay puntos en que el OD alcanza niveles muy bajos, como el punto 14, donde se unen corrientes del estero Mozapan, del Canal de Navegación, arroyo Correa y Laguna de Préstamos (3.31 mg/L); y el punto 16, en el arroyo Apepecho, que tiene influencia de una corriente de agua acumulada en época de lluvias proveniente de la presa Agua de Minas, la cual es neutralizada con $\mathrm{MgO}$ antes de ser vertida al arroyo $(4.47 \mathrm{mg} / \mathrm{L})$. La presa 


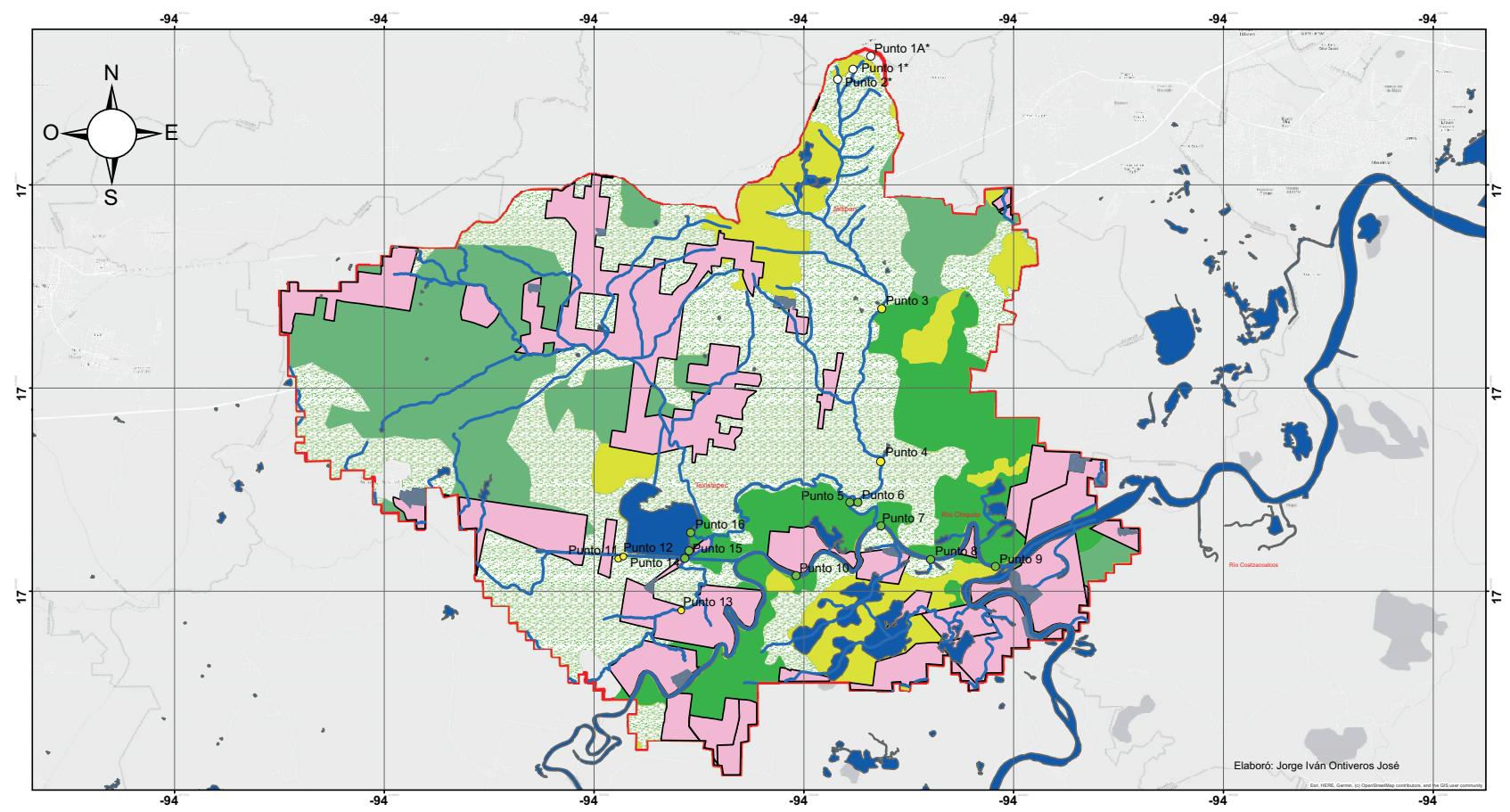

ICA obtenidos mediante la aplicación de la metodología NSF

\begin{tabular}{|c|c|c|c|c|c|c|c|c|}
\hline \multirow{5}{*}{$\begin{array}{l}\text { Marco de Referencia: } \\
\text { Coordenadas UTM } \\
\text { Datum WGS84 } \\
\text { Zona } 15 \text { Norte }\end{array}$} & & & \multirow{6}{*}{$\begin{array}{l}\text { Puntos de Muestreo } \\
\text { ○ Buena } \\
\text { ○ Medianamente Contaminada } \\
\text { ○ No Aplica }\end{array}$} & \multirow{5}{*}{$\begin{array}{l}\text { Cuerpos de Agua } \\
\text { Zonas Parceladas } \\
\text { Agricultural de Temporal Anual } \\
\text { Agricultura de Humedad Anual }\end{array}$} & \multirow{5}{*}{$\begin{array}{l}\text { Asentamientos Humanos } \\
\text { Zona Urbana } \\
\text { Área de Estudio }\end{array}$} & \multicolumn{3}{|c|}{ Escala de clasificación del ICA-NSF } \\
\hline & Escala: & Fech & & & & CALIDAD & RANGO & ESCALA DE COLOR \\
\hline & 1:50000 & Junio 2020 & & & & Excelente & $91-100$ & Azul \\
\hline & Fuente & & & & & Buena & $71-90$ & Verde \\
\hline & $\begin{array}{l}\text { INEGI, SI } \\
\text { CONAPO }\end{array}$ & $\begin{array}{l}\text { IATL, RAN, } \\
\text { Py CONABIO }\end{array}$ & & & & $\begin{array}{l}\text { Media } \\
\text { Mala }\end{array}$ & $\begin{array}{l}51-70 \\
26-50\end{array}$ & $\begin{array}{l}\text { Amarillo } \\
\text { Narania }\end{array}$ \\
\hline 1.5 & 6 & 9 & & Pastizal Cultivado & & Muy Mala & $0-25$ & Rojo \\
\hline
\end{tabular}

Fig. 4. ICA obtenidos mediante la aplicación de la metodología NSF.

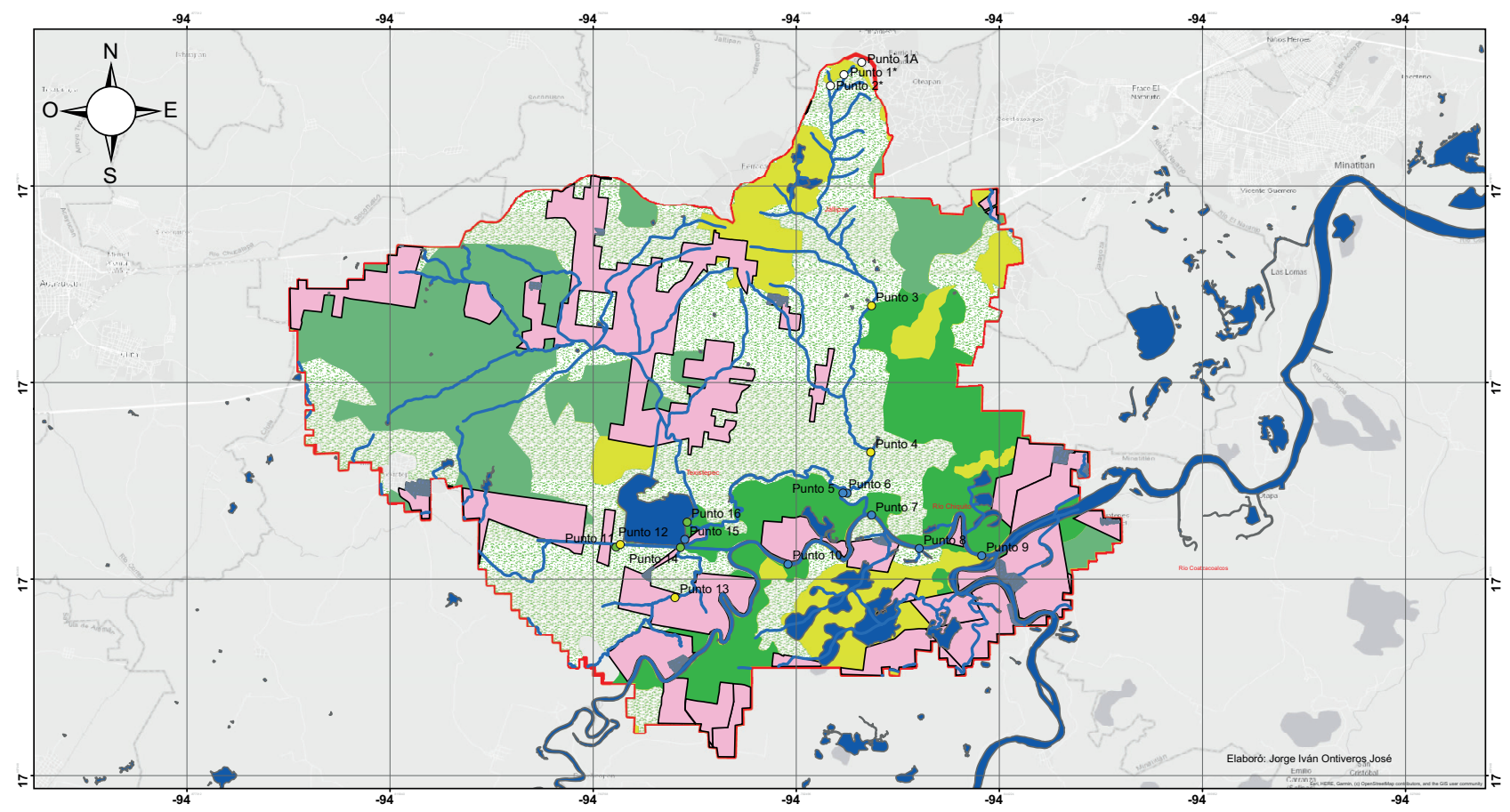

ICA obtenidos mediante la aplicación de la metodología CNA
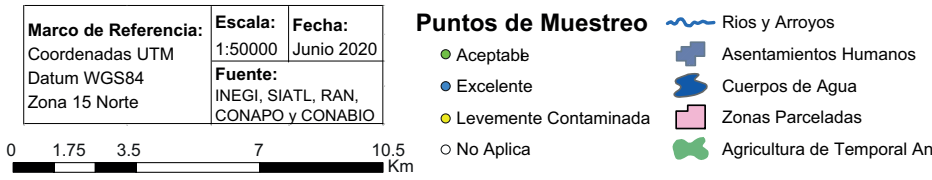

Agricultura de Humedad Anual

Pastizal Cultivado

-1- Zona Urbana

$\square$ Área de Estudio

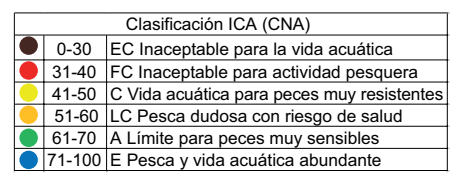

Fig. 5. ICA obtenidos mediante la aplicación de la metodología CNA. 
CUADRO IX. LÍMITES DE REFERENCIA PARA PARÁMETROS IMPORTANTES EN EL AGUA (USO PARA PROTECCIÓN DE LA VIDA ACUÁTICA).

\begin{tabular}{|c|c|c|c|}
\hline Párametro & Unidad & Rango & Referencias \\
\hline $\mathrm{pH}$ & & $6.5-8.5$ & $\begin{array}{l}\text { Normas de calidad del agua superficial para Moldavia. Clase II* } \\
\text { OECD (2007) }\end{array}$ \\
\hline Temperatura & ${ }^{\circ} \mathrm{C}$ & $20-40$ & NOM-001-SEMARNAT-1996 \\
\hline Oxígeno Disuelto & $\mathrm{mg} / \mathrm{L}$ & $\geq 5$ & Criterios Ecológicos de Calidad del Agua CE-CCA-001/89 \\
\hline Conductividad Específica & $\mu \mathrm{S} / \mathrm{cm}$ & $150-200$ & $\begin{array}{l}\text { Norma de calidad ambiental y de descarga de efluentes: Recurso agua. } \\
\text { Presidencia de la República de Ecuador (2002) }\end{array}$ \\
\hline Sólidos Disueltos Totales & $\mathrm{mg} / \mathrm{L}$ & $120-600$ & $\begin{array}{l}\text { Norma de calidad ambiental y de descarga de efluentes: Recurso agua. } \\
\text { Presidencia de la República de Ecuador (2002) }\end{array}$ \\
\hline $\begin{array}{l}\text { Sólidos Suspendidos } \\
\text { Totales }\end{array}$ & $\mathrm{mg} / \mathrm{L}$ & $25 \leq \mathrm{SST} \leq 75$ & CONAGUA (2010) \\
\hline $\mathrm{DBO}_{5}$ & $\mathrm{mg} / \mathrm{L}$ & 5 & $\begin{array}{l}\text { Normas de calidad del agua superficial para Moldavia. Clase II* } \\
\text { OECD (2007) }\end{array}$ \\
\hline DQO & $\mathrm{mg} / \mathrm{L}$ & 7 & $\begin{array}{l}\text { Normas de calidad del agua superficial para Moldavia. Clase II* } \\
\text { OECD (2007) }\end{array}$ \\
\hline Fósforo Total & $\mathrm{mg} \mathrm{PO}_{4}^{-2} / \mathrm{L}$ & $\begin{array}{l}\leq 0.1 \\
<0.010\end{array}$ & $\begin{array}{l}\text { Criterios Ecológicos de Calidad del Agua CE-CCA-001/89. Clase I** } \\
\text { ECE (1993) } \\
\text { UNECE (1994) Clase I** }\end{array}$ \\
\hline$\underline{\mathrm{N}-\mathrm{NH}_{4}}{ }^{+}$ & $\mathrm{mg} / \mathrm{L}$ & 0.06 & Criterios Ecológicos de Calidad del Agua CE-CCA-001/89 \\
\hline $\mathrm{N}-\mathrm{NO}_{3}{ }^{-}$ & $\mathrm{mg} / \mathrm{L}$ & 3 & $\begin{array}{l}\text { Normas de calidad del agua superficial para Moldavia. Clase II* } \\
\text { OECD (2007) }\end{array}$ \\
\hline
\end{tabular}

*La calidad del agua correspondiente a esta clase permite los siguientes usos: funcionamiento de los ecosistemas, cría/protección de peces, recreación, irrigación, uso industrial del agua, generación de energía, extracción de minerales y transporte.

**Agua clara, oligotrófica con, a lo sumo, contaminación antrópica muy leve y ocasional con materia orgánica. Bajo contenido en nutrientes, proporciona zonas de desove para los salmónidos.

Agua de Minas es una zona inundable conectada al arroyo Apepecho, que en el pasado fue una laguna que recibió agua ácida e hidrocarburo generados durante la extracción de azufre en una antigua unidad minera que actualmente es un pasivo ambiental; por tal motivo, el efluente de la presa es neutralizado con $\mathrm{MgO}$. A pesar de los bajos niveles de OD en los puntos 14 y 16, los índices del NSF y la CNA catalogan las aguas como de calidad buena y aceptable, respectivamente; en el resto de los puntos $(3,4,11,12$ y 13) el OD fluctuó de 0 a $3.26 \mathrm{mg} / \mathrm{L}$, lo que en ambas metodologías se refleja en una clasificación de la calidad en el nivel de agua contaminada.

Es importante considerar que la zona de los puntos 11 a 16 recibe la influencia de escorrentías procedentes del cultivo de árboles de palma de aceite, maíz y chile, entre otros, aunque por ser periodo de estiaje el impacto sería menor.

\section{pH}

$\mathrm{El} \mathrm{pH}$ es un factor importante en todos los procesos biológicos que ocurren en las aguas superficiales. Dentro de sus lineamientos sobre calidad del agua, la OECD (2007) recomienda un rango de 6.5 a 8.5
(Cuadro IX). En esta investigación, dicho rango únicamente se cumple en los puntos 3, 4, 5, 8 y 9, en tanto que en el resto de los puntos el pH fluctuó de 5.81 a 6.43 .

\section{Sólidos suspendidos totales (SST) y turbidez}

La cantidad de luz disponible a diferentes profundidades en los lagos y ríos es importante para la ecología del cuerpo de agua. El agua misma, las sustancias disueltas y la materia particulada absorben luz visible, reduciéndose la luz de una determinada longitud de onda a medida que la profundidad se incrementa (Heinke y Henry 1999). La turbidez es un parámetro indicativo de la probable reducción de la fotosíntesis algácea, y tiene una estrecha relación con la presencia de SST. El incremento en los niveles de SST en los cuerpos de agua provoca turbidez y reduce la penetración de luz solar, impidiendo el desarrollo de la vegetación acuática y afectando al resto de la biodiversidad.

En este sentido, CONAGUA (2010) estableció una escala de clasificación de la calidad del agua superficial con base en SST. Un rango de $25<$ $\mathrm{SST} \leq 75$ (en $\mathrm{mg} / \mathrm{L})$ se cataloga como bueno, ya 
que favorece la conservación de comunidades acuáticas. En todos los puntos de muestreo se cumplió con el rango superior de SST $(75 \mathrm{mg} / \mathrm{L})$, registrándose valores incluso menores a $25 \mathrm{mg} / \mathrm{L}$ en algunos puntos.

\section{Conductividad específica y sólidos disueltos totales (SDT)}

Dos parámetros fuertemente ligados e importantes en la vida acuática son los SDT y la conductividad específica. Espinal-Carreón et al. (2013) indican que en épocas de sequía la capacidad de dilución de los ecosistemas acuáticos es baja, lo que puede incrementar su deterioro al aumentar los tiempos de retención en lagos, embalses y humedales; las épocas de sequía implican un aumento en la temperatura del agua y la salinidad, lo que causa un deterioro generalizado de la calidad del agua.

Algunas especies son especialmente sensibles a las sales, cuyas concentraciones muy bajas o altas pueden afectar la homeoestasis en su medio interno en ambientes dulceacuícolas (Blancas-Arroyo et al. 2014). La Norma de calidad ambiental y de descarga de efluentes de Ecuador (Presidencia de la República de Ecuador 2002) establece, para uso del agua en pesca, un rango de conductividad específica de 150-200 $\mu \mathrm{s} / \mathrm{cm}$ y de $120-600 \mathrm{mg} / \mathrm{L}$ para SDT. Respecto a la conductividad específica, se rebasó el límite máximo en todos los puntos de muestreo. Con relación a la concentración de SDT, en los puntos de muestreo $3,4,12,13$ y 16 se ubicó en el rango recomendado, pero en los puntos 5 al 11, 14 y 15 sus valores fueron menores a $120 \mathrm{mg} / \mathrm{L}$.

\section{$\mathrm{DBO}_{5}$ y DQO}

Si se analizan los llamados parámetros de presión ecológica como la $\mathrm{DBO}_{5}$ y la DQO, cuya disparidad en los datos obtenidos en esta investigación llama la atención, la OECD (2007) recomienda límites máximos recomendables de 5 y $7 \mathrm{mg} / \mathrm{L}$, respectivamente, en aguas superficiales para protección de la vida acuática. En la mayoría de los puntos se rebasan estos criterios.

De los límites máximos recomendados por la OECD (2007), se deduce que una relación óptima $\mathrm{DBO}_{5} / \mathrm{DQO}$ es igual a 0.7 , aproximadamente. Se calcularon las relaciones $\mathrm{DBO}_{5} / \mathrm{DQO}$ de los puntos 3 al 16 , las cuales se muestran en el cuadro $X$. Se observa que la relación $\mathrm{DBO}_{5} / \mathrm{DQO}$ tuvo un valor mínimo de 0.0083 en el punto 7 y un valor máximo de 0.1421 en el punto 3. Evidentemente, todos los valores obtenidos están por debajo de la relación 0.5 (y mucho más de una relación óptima de 0.7), lo cual indica que el consumo de oxígeno en el cuerpo de agua está
CUADRO X. RELACIONES DBO 5 /DQO EN CADA PUNTO DE MUESTREO.

\begin{tabular}{cc}
\hline Punto de muestreo & Relación $\mathrm{DBO}_{5} / \mathrm{DQO}$ \\
\hline 3 & 0.1421 \\
4 & 0.0231 \\
5 & 0.0504 \\
6 & 0.0309 \\
7 & 0.0083 \\
8 & 0.0752 \\
9 & 0.0696 \\
10 & 0.0542 \\
11 & 0.0420 \\
12 & 0.0420 \\
13 & 0.0419 \\
14 & 0.0408 \\
16 & 0.0419 \\
\hline
\end{tabular}

relacionado con compuestos de difícil degradación como residuos de plaguicidas o cualquier otro tipo de residuo industrial. No obstante, debido a que la DQO no está considerada en ninguna de las metodologías aplicadas, no afecta los valores del ICA.

Es cuestionable que parámetros como la $\mathrm{DBO}_{5}$ rebasen criterios recomendados y esto no impacte la evaluación de la calidad del agua en la aplicación de las metodologías utilizadas; peor aún, que no se incluyan parámetros como la DQO que presentaron valores indicativos de contaminación por materia orgánica difícilmente biodegradable.

Resultados similares fueron reportados por Caho-Rodríguez y López-Barrera (2017), quienes evaluaron la calidad del agua del sector occidental del humedal Torca-Guaymaral en Colombia. Aunque un humedal tiene un comportamiento hidrológico muy diferente a un arroyo o un río, sirve de referencia para establecer la naturaleza de los contaminantes que pueden estar presentes en un cuerpo de agua.

\section{Fósforo total}

El fósforo ingresa en el sistema acuático de forma natural debido a la lixiviación de las rocas (en las cuales está presente como fosfato de calcio) y al transporte de nutrientes desde los suelos. Generalmente se encuentra en cantidades limitadas en ríos y lagos, reduciéndose aún más su concentración debido al metabolismo de animales y vegetales, ya que es un elemento indispensable. Cuando se detectan concentraciones elevadas de este elemento en fuentes de agua, se asocian al aporte de actividades humanas como la agricultura y vertidos domésticos e industriales (Kiely 1999). 
En la revisión de normatividad internacional sobre límites recomendados para fósforo en aguas superficiales, se encontró que la UNECE (1994) establece una concentración de fósforo total en ríos y arroyos no mayor de $0.01 \mathrm{mg} / \mathrm{L}$. De acuerdo con esta normatividad, los puntos $3,4,5,8,12,14,15$ y 16 rebasan el límite recomendado; en contraste, el valor recomendado en los Criterios Ecológicos de Calidad del Agua CE-CCA-001/89 (SEDUE 1989) es de $0.1 \mathrm{mg} / \mathrm{L}$ de $\mathrm{PO}_{4}{ }^{=}$, valor que sólo se sobrepasa en el punto 4. En condiciones de bajo OD, el crecimiento de los organismos vegetales es reducido, lo que ocasiona que los niveles de fósforo aumenten (tal como sucedió en los puntos 3, 4, 12, 14 y 16, si se considera el límite máximo de $0.01 \mathrm{mg} / \mathrm{L}$ recomendado por UNECE [1994]). Cuando se presentan mayores condiciones oxidantes, el fósforo queda ligado a los sedimentos, reduciéndose su concentración en la columna de agua, caso observado en algunos puntos de muestreo en esta investigación (Heinke y Henry 1999).

La ponderación que aportan ambas metodologías con relación al fósforo no afecta en gran medida la evaluación de la calidad del agua en otros puntos, por lo que en sitios donde hay evidencia de procesos de eutrofización, como la Laguna de Préstamos (con $0.015 \mathrm{mg} / \mathrm{L}$ de $\mathrm{PO}_{4}{ }^{\prime}$, punto 15 ), el agua se clasifica como de buena y excelente calidad. Algo similar ocurre en otros puntos de muestreo, donde se encontraron evidencias de eutrofización (14 y 16) y el agua se clasifica como de calidad buena a aceptable.

\section{Nitrógeno}

Los vertidos domésticos y sanitarios son fuentes principales de nitrógeno en el agua. Es importante mencionar que los procesos de metabolización del nitrógeno orgánico y del $\mathrm{N}_{-} \mathrm{NH}_{4}{ }^{+}$se ven favorecidos por niveles de OD mayores a $0.3 \mathrm{mg} / \mathrm{L} \mathrm{y} \mathrm{pH}$ de 6 a 9.6 (Madigan et al. 2010). En este sentido, el $\mathrm{N}-\mathrm{NH}_{4}{ }^{+}$superó los límites máximos recomendados (0.06 mg/L, CE-CCA-001/89) en todos los puntos. $\mathrm{El} \mathrm{pH}$ no fue un problema significativo en tanto que, como ya se indicó, el OD alcanzó en algunos puntos valores por debajo de $5 \mathrm{mg} / \mathrm{L}$, pero dentro del intervalo necesario para la metabolización de ambas formas de nitrógeno. Nuevamente, esto podría sugerir que el oxígeno se consumió en la oxidación de compuestos de difícil degradación.

Es importante mencionar que el modelo NSF no considera el nitrógeno en forma de $\mathrm{N}_{-} \mathrm{NH}_{4}{ }^{+}$, sino como ${\mathrm{N}-\mathrm{NO}_{3}}^{-}$. En este sentido, la OECD (2007) establece un límite máximo permisible de $3 \mathrm{mg} / \mathrm{L}$ de $\mathrm{N}-\mathrm{NO}_{3}{ }^{-}$en aguas superficiales para cría y protección de peces, nivel que no se vio rebasado en ninguno de los puntos de muestreo.

La eutrofización, uno de los problemas de calidad del agua más significativos, provoca graves perjuicios a la actividad pesquera debido a cambios en las especies de peces presentes en los cuerpos de agua superficial, como resultado de niveles bajos de OD en las aguas inferiores.

\section{Otros parámetros considerados en el cálculo del ICA-CNA}

Los parámetros que se consideraron en la metodología ICA-CNA, además de los ya contemplados en la metodología NSF y los ya discutidos (como la conductividad específica y los SST), son: cloruros, alcalinidad y dureza total. A este respecto, los Criterios Ecológicos de Calidad del Agua CE-CCA-001/89 (SEDUE 1989) recomiendan un valor máximo en cloruros de $250 \mathrm{mg} / \mathrm{L}$, para el cual no hay problema, ya que los valores en este parámetro tuvieron un mínimo y un máximo de 8.93 y $87.49 \mathrm{mg} / \mathrm{L}$, respectivamente. La alcalinidad varió en el rango de 10 a $275 \mathrm{mg} / \mathrm{L}$, lo cual es indicativo del poder de amortiguamiento del agua, es decir, su capacidad para amortiguar cambios en los valores del pH. UNECE (1994) recomienda un valor superior a $200 \mathrm{mg} / \mathrm{L}$, para que la capacidad de amortiguamiento del agua sea adecuada. En este sentido, el punto 12 es el único que cumple con este criterio, por lo que el resto de los puntos carece de un poder óptimo de amortiguamiento.

Con relación a la dureza del agua, Rubio-Arias et al. (2014) reportan un rango recomendable para la vida acuática de 100 a $150 \mathrm{mg} / \mathrm{L}$. Los valores registrados fluctuaron entre 14 y $217.2 \mathrm{mg} / \mathrm{L}$, quedando fuera del intervalo de referencia.

\section{CONCLUSIONES}

De acuerdo con la metodología de la NSF, la calidad del agua en los 13 puntos de muestreo se clasificó como medianamente contaminada a buena, en tanto que según el modelo de la CNA la categorización fue levemente contaminada, aceptable y excelente. Los parámetros que más afectan la calidad del agua por incumplir con los criterios recomendados son OD, fósforo total y N-NH${ }_{4}^{+}$.

La DQO varió en el rango de 105.66 a 442.85 $\mathrm{mg} / \mathrm{L}$, valores que alcanzan niveles similares a los encontrados en aguas residuales urbanas (o industriales). La escala de clasificación de la calidad del agua conforme a la DQO (CONAGUA 2010) cataloga el agua de los 13 puntos muestreados como contami- 
nada a fuertemente contaminada. Por otra parte, los valores de $\mathrm{DBO}_{5}$ fluctuaron de 2.42 a $18.16 \mathrm{mg} / \mathrm{L}$, clasificando las aguas como de calidad aceptable a excelente, valores que no se comparan con los de DQO, lo que pudiera ser indicativo de la descarga de vertidos que contienen compuestos de difícil degradación biológica.

El criterio de la DQO indica la presencia de contaminantes de difícil degradación provenientes de descargas industriales o de la aplicación de plaguicidas y herbicidas en los campos de cultivo, los cuales pudieran estar presentes en las aguas superficiales estudiadas causando estragos a la vida de ciertas especies acuáticas.

Independientemente de los resultados obtenidos con ambas metodologías, es necesario considerar en la evaluación de la calidad del agua parámetros indicativos de la presencia de sustancias difícilmente biodegradables como la DQO o tóxicas, dependiendo de las circunstancias específicas de la zona de estudio; asimismo, es importante considerar criterios o rangos recomendables para los diversos parámetros, como lo han hecho diversos investigadores (CCME 2012, González-Acevedo et al. 2016, Mena-Mejía et al. 2017). Por último, al incluir parámetros relevantes para evaluar la calidad del agua es conveniente hacerlo considerando criterios o rangos de calidad de acuerdo con las normativas nacionales e internacionales.

\section{AGRADECIMIENTOS}

Se agradece al Tecnológico Nacional de México por el soporte financiero para el desarrollo del proyecto 6518.18-P, Apoyo a la investigación científica y tecnológica. Asimismo, se agradece a las S.C.P.P. Estero Mozapan y Río Chiquito, Afluentes y Anexos, La Lajilla El Palmar, S.C. de R.L., Paso el Moral S. C. de R.L. y Sembradores de Aztapan, por su apoyo en la realización de los muestreos en la zona de estudio.

\section{REFERENCIAS}

APHA-AWWA-WPCF (1992). Métodos normalizados para el análisis de aguas potables y residuales. $17^{\mathrm{a}} \mathrm{ed}$. Ediciones Díaz de Santos, Madrid, España, 1830 pp.

Asadollahfardi G. (2015). Water quality management assessment and interpretation. Springer, Heidelberg, New York, Dordrecht, London. 124 pp. https://doi. org/10.1007/978-3-662-44725-3

Azpilcueta-Pérez M.E., Pedroza-Sandoval A., Sánchez-
Cohen I., Salcedo-Jacobo M.R. y Trejo-Calzada R. (2017). Calidad química del agua en un área agrícola de maíz forrajero (Zea mays L.) en la Comarca Lagunera, México. Rev. Int. Contam. Ambie. 33 (1), 75-83. https://doi.org/10.20937/RICA.2017.33.01.07

Balmaseda-Espinosa C. y García-Hidalgo Y. (2014). Índice canadiense de calidad de las aguas para la cuenca del río Naranjo, provincia Las Tunas, Cuba. Revista Ciencias Técnicas Agropecuarias 23 (3), 11-16.

Batabyal A.K. y Chakraborty S. (2015). Hydrogeochemistry and water quality index in the assessment of groundwater quality for drinking uses. Water Environ. Res. 87 (7), 607-617. https://doi.org/10.2175/106143 015X14212658613956

Blancas-Arroyo G.A., Frías-Sevilla R., de la RosaPimental E., Suárez-Navarro V., Castro-Gómez J.R. y Magaña-Morales J. (2014). Efecto de la salinidad en la sobrevivencia de peces silvestres del género Chirostoma durante el transporte y mantenimiento en laboratorio. Hidrobiológica 24 (3), 223-230.

Brown R.M., McClelland N.I., Deininger R.A. y Tozer R.G. (1970). A water quality index - do we dare? Water \& Sewage Works 117 (10), 339-343.

Caho-Rodríguez C.A. y López-Barrera E.A. (2017). Determinación del índice de calidad de agua para el sector occidental del humedal Torca-Guaymaral empleando las metodologías UWQI y CWQI. Producción + Limpia 12 (2), 35-49. https://doi.org/10.22507/pml.v12n2a3

CCME (2012). Application and testing of the water quality index in Atlantic Canada. Report summary. Canadian Council of Ministers of the Environment [en línea]. https://www.ccme.ca/files/Resources/water/ water_quality/awi_en_posting.pdf 27/07/2019

CONAGUA (2010). Estadísticas del agua en México edición 2010. Comisión Nacional del Agua, México [en línea]. http://www.conagua.gob.mx/CONAGUA07/Publicaciones/Publicaciones/EAM201016Junio2010.pdf 14/06/2020

CONAGUA (2019). Calidad del agua en México. Comisión Nacional del Agua, México [en línea]. https:// www.gob.mx/conagua/articulos/calidad-del-agua 27/07/2019

De la Mora-Orozco C., Flores-Garnica J.G., Ruiz-Corral J.A. y García-Velasco J. (2004). Modelaje estocástico de la variabilidad espacial de la calidad de agua en un ecosistema lacustre. Rev. Int. Contam. Ambie. 20 (3), 99-108.

Espinal-Carreón T., Sedeño-Díaz J.E. y López-López E. (2013). Evaluación de la calidad de agua en la laguna de Yuriria, Guanajuato, México, mediante técnicas multivariadas: un análisis de valoración para dos épocas: 2005, 2009-2010. Rev. Int. Contam. Ambie. 29 (3), 147-163. 
Espejo L., Kretschmer N., Oyarzún J., Meza F., Nuñez J., Maturana H., Soto G., Oyarzo P., Garrido M., Suckel F., Amezaga J. y Oyarzún R. (2012). Application of water quality indices and analysis of the surface water quality monitoring network in semiarid north-central Chile. Environ. Monit. Assess. 184 (9), 5571-5588. https://doi.org/10.1007/s10661-011-2363-5

Fernández N. y Solano F. (2005). Índices de calidad y de contaminación del agua. Ed. Java, Universidad de Pamplona, España, 142 pp.

González-Acevedo Z.I., Padilla-Reyes D.A. y RamosLeal J.A. (2016). Quality assessment of irrigation water related to soil salinization in Tierra Nueva, San Luis Potosí, Mexico. Revista Mexicana de Ciencias Geológicas 33 (3), 271-285.

Gracia C.E. (2004). Evaluación y clasificación de las aguas del río San Antonio. Tesis de Licenciatura. Facultad de Ciencias Químicas, Universidad Veracruzana, Orizaba, Veracruz, México, 75 pp.

Guzmán-Colis G., Thalasso F., Ramírez-López E.M., Rodríguez-Narciso S., Guerrero-Barrera A.L. y AvelarGonzález F.J. (2011). Evaluación espacio-temporal de la calidad del agua del río San Pedro en el estado de Aguascalientes, México. Rev. Int. Contam. Ambie. 27 (2), 89-102.

Heinke G.W. y Henry J.G. (1999). Ingeniería ambiental. $2^{\mathrm{a}}$ ed. Pearson Educación, México, 778 pp.

House M.A. y Ellis J.B. (1987). The development of water quality indices for operational management. Wat. Sci. Tech. 19 (9), 145-154. https://doi.org/10.2166/ wst. 1987.0076

Kiely G. (1999). Ingeniería ambiental. Fundamentos, entornos, tecnologías y sistemas de gestión. McGrawHill, Madrid, España, 1331 pp.

López-Álvarez B., Ramos-Leal J.A., Santacruz G., MoránRamírez J., Carranco-Lozada S.E., Noyola-Medrano M.C. y Pineda-Martínez L.F. (2013). Cálculo del índice de pobreza del agua en zonas semiáridas: caso Valle de San Luis Potosí. Rev. Int. Contam. Ambie. 29 (4), 249-260.

Mădălina P. y Breabăn I.G. (2014). Water quality index - An instrument for water resources management. Ponencia [en línea]. https://www.researchgate.net/ publication/266737680_WATER_QUALITY_INDEX_-_AN_INSTRUMENT_FOR_WATER_RESOURCES_MANAGEMENT 19/06/2020

Madigan M.T, Martinko J.M. y Parker J. (2010). Brock. Biología de los microorganismos. $10^{\mathrm{a}}$ ed. Pearson Educación, Nueva Jersey, EUA, 1089 pp.

Mena-Mejía I., Bustamante-González Á., Vargas-López S., Olvera-Hernández J.I. y Méndez-Espinoza J.A. (2017). Evaluación de la condición ecológica del río Zahuapan. Rev. Int. Contam. Ambie. 33 (1), 7-19. https://doi.org/10.20937/RICA.2017.33.01.01
Miller R.R. (2009). Peces dulceacuícolas de México. Comisión Nacional para el Conocimiento y Uso de la Biodiversidad, México, 180 pp.

Mosquera-Murillo Z. y Mosquera-Mosquera M.M. (2017). Diversidad de la entomofauna acuática y calidad de agua en quebradas del río San Juan, Chocó - Colombia. Rev. U.D.C.A. Act. \& Div. Cient. 20 (1), 149-161.

NAS (1975). Planning for environmental indices. A report of the Planning Committee on Environmental Indices. Reporte técnico. National Academy of Science, Washington, DC, EUA, 47 pp.

NSF (2019). Consumer resources: Water Quality Index (WQI). National Sanitation Foundation [en línea]. www. nsf.org/consumer/just_for_kids/wqi.asp 13/07/2019

OECD (2007). EAP Task Force proposed system of surface water quality standards for Moldova. Reporte técnico. Organisation for Economic Cooperation and Development, París, Francia, 49 pp.

Olguín E.J., González-Portela R.E., Sánchez-Galván G., Zamora-Castro J.E. y Owen T. (2010). Contaminación de ríos urbanos: El caso de la subcuenca del río Sordo en Xalapa, Veracruz, México. Rev. Latinoam. Biotecnol. Amb. Algal. 1 (2),178-190.

Ott W.R. (1978). Environmental indices: Theory and practice. Ann Arbor Science Publishers, Michigan, EUA, 348 pp.

Presidencia de la República de Ecuador (2002). Norma de calidad ambiental y de descarga de efluentes: Recurso agua. Environmental Law Alliance Worldwide [en línea]. https://www.elaw.org/ content/ecuador-normade-calidad-ambiental-y-de-descarga-de-efluentesrecurso-agua 14/06/2020

Quiroz-Fernández L.S., Izquierdo-Kulich E. y MenéndezGutiérrez C. (2017). Aplicación del índice de calidad de agua en el río Portoviejo, Ecuador. Ingeniería Hidráulica y Ambiental XXXVIII (3), 41-51.

Reza R. y Singh G. (2010). Assessment of ground water quality status by using water quality index method in Orissa, India. World Appl. Sci. J. 9 (12), 1392-1397.

Rubio-Arias H., Contreras-Caraveo M., Quintana R.M., Saucedo-Terán R.A. y Pinales-Munguía A. (2012). An overall water quality index (WQI) for a man-made aquatic reservoir in Mexico. Int. J. Environ. Res. Public Health. 9 (5), 1687-1698. https://doi.org/10.3390/ ijerph905 1687

Rubio-Arias H.O., Ortiz-Delgado R.C., Quintana-Martínez R.M., Saucedo-Terán R.A, Ochoa-Rivero J.M. y Rey-Burciaga N.I. (2014). Índice de calidad de agua (ICA) en la presa La Boquilla en Chihuahua, México. Ecosistemas y Recursos Agropecuarios 1 (2), 139-150.

Samboni-Ruiz N.E., Carvajal-Escobar Y. y Escobar J.C. (2007). Revisión de parámetros fisicoquímicos como 
indicadores de calidad y contaminación del agua. Revista Ingeniería e Investigación 27 (3), 172-181.

SECOFI (1980). Norma Mexicana NMX-AA-014-1980. Cuerpos receptores. Muestreo. Secretaría de Comercio y Fomento Industrial. Diario Oficial de la Federación, México, 5 de septiembre.

SEDUE (1989). Acuerdo por el que se establecen los Criterios Ecológicos de Calidad del Agua CE-CCA-001/89. Secretaría de Desarrollo Urbano y Ecología. Diario Oficial de la Federación, México, 13 de diciembre.

SEMARNAT (2019). Consulta temática sobre indicadores de calidad del agua. Gerencia de Calidad del Agua. Secretaría de Medio Ambiente y Recursos Naturales [en línea]. http://dgeiawf.semarnat.gob.mx:8080/ibi_apps/ WFServlet?IBIF ex=D3 R AGUA05 01\&IBIC user $=$ dgeia_mce $\&$ IBIC_pass $=$ dgeia_mce $14 / 06 / 2020$
Tyagi S., Sharma B., Singh P. y Dobhal R. (2013). Water quality assessment in terms of water quality index. American Journal of Water Resources 1 (3), 34-38. https://doi.org/10.12691/ajwr-1-3-3

UNECE (1994). Standard statistical classification of surface freshwater quality for the maintenance of aquatic life. En: Reading in international environment statistics. United Nations Economic Commission for Europe, New York, NY, USA, 1994, 543 pp.

Yogendra K. y Puttaiah E.T. (2008). Determination of water quality index and suitability of an urban waterbody in Shimoga Town, Karnataka. Memorias. The 12th World Lake Conference: Taal 2007. Jaipur, Rajasthan, India. 28 de octubre-2 de noviembre 2007,. 342-346. 